\title{
NORMAL APPROXIMATION FOR RANDOM SUMS
}

\author{
A. D. BARBOUR, ${ }^{*}$ Universität Zürich \\ AIHUA XIA,** The University of Melbourne
}

\begin{abstract}
In this paper, we adapt the very effective Berry-Esseen theorems of Chen and Shao (2004), which apply to sums of locally dependent random variables, for use with randomly indexed sums. Our particular interest is in random variables resulting from integrating a random field with respect to a point process. We illustrate the use of our theorems in three examples: in a rather general model of the insurance collective; in problems in geometrical probability involving stabilizing functionals; and in counting the maximal points in a two-dimensional region.
\end{abstract}

Keywords: Stein's method; Berry-Esseen bound; point process; random field; local dependence; two-dimensional maximum

2000 Mathematics Subject Classification: Primary 60F05

Secondary 60G50; 60G57; 60G60

\section{Introduction}

Of the techniques available for establishing the accuracy of approximation in the central limit theorem for sums of dependent random variables, Stein's (1972) method has become one of the most popular. It readily delivers error bounds which are often of or close to the correct asymptotic order, when the distance between distributions is measured with respect to the (bounded) Wasserstein distance; see, for example, Erickson (1974) and Barbour et al. (1989). If a bound for the error in Kolmogorov distance, $d_{\mathrm{K}}$, is preferred (where, for two probability measures $P$ and $Q$ on $\left.\mathbb{R}, d_{\mathrm{K}}(P, Q):=\sup _{x}|P(-\infty, x]-Q(-\infty, x]|\right)$, the arguments needed are more involved, but there have nonetheless been notable successes, such as Bolthausen's (1984) Berry-Esseen bound for the combinatorial central limit theorem. More recently, Baldi and Rinott (1989) used a theorem of Stein (1986, p. 35) to establish rates of convergence for sums of dependent random variables in terms of properties of an associated dependency graph. Even though the rates obtained were not optimal, even for bounded summands, their theorem has proved extremely useful. This approach has been substantially refined, for example in Dembo and Rinott (1996) and, for multivariate random variables, Rinott and Rotar (1996); however, except for bounded summands, the correct rate of convergence could not usually be attained.

In a recent paper, Chen and Shao (2004) have used the concentration inequality approach to Stein's method to establish accurate Berry-Esseen bounds for sums, $W=\sum_{i=1}^{n} X_{i}$, of centred

\footnotetext{
Received 21 March 2005; revision received 2 May 2006.

* Postal address: Institut für Mathematik, Universität Zürich, Winterthurerstrasse 190, 8057 Zürich, Switzerland. Email address: adb@amath.unizh.ch

Partially supported by Schweizerischer Nationalfondsprojekt Nr. 20-67909.02.

** Postal address: Department of Mathematics and Statistics, The University of Melbourne, Parkville, VIC 3010, Australia. Email address: xia@ms.unimelb.edu.au

Partially supported by the ARC Centre of Excellence for Mathematics and Statistics of Complex Systems.
} 
random variables, under a variety of local dependence assumptions. In particular, in their Theorem 2.4, the error bound is expressed very simply in Lyapounov form, being of order

$$
O\left(\kappa^{p-1} \sum_{i=1}^{n} \mathrm{E}\left|X_{i}\right|^{p}(\operatorname{var} W)^{-p / 2}\right)
$$

for $2<p \leq 3$. Here, $\kappa:=\max _{i} \operatorname{card}\left(N\left(C_{i}\right)\right)$ for an index set, $N\left(C_{i}\right)$, corresponding to an extended dependence neighbourhood of $X_{i}$; see condition (LD4) below. Their bound promises to find wide application.

In this paper, we are concerned with modifying the theory of Chen and Shao (2004) in order to apply it to randomly indexed sums. The topic of randomly stopped (partial sum) processes can be traced back to Anscombe (1952) and Rényi (1960), and there is now a substantial theory (see, for example, Gnedenko and Korolev (1996), Silvestrov (2004), and Kläver and Schmitz (2006)). Our interest is rather in having as random index set the points of a point process, which may also (locally) influence the values of the summands. (In the literature, the term 'point field' is also occasionally used instead of point process; see Stoyan and Stoyan (1994).) More precisely, we wish to re-express the theorems of Chen and Shao (2004) in such a way that they can be directly applied to random variables of the form $W=\int_{\Gamma} F_{\alpha} H(\mathrm{~d} \alpha)$, where $H$ is a point process on a locally compact, second-countable Hausdorff topological space $\Gamma$ with locally finite mean measure, $F_{\alpha}$ is a random field, and the signed measure with density $F_{\alpha} H(\mathrm{~d} \alpha)$ satisfies some local dependence hypotheses. (A measure is locally finite if it has finite measure on every relatively compact set.) For example, $H$ might be a Poisson process and we might have $F_{\alpha}=\mathbf{1}_{\{H(B(\alpha, \rho) \backslash\{\alpha\})=0\}}$ for some $\rho>0$, where $B(\alpha, \rho)$ denotes the closed ball around $\alpha$ with radius $\rho$; in this case, $W$ counts the $\rho$-isolated points of $H$ (cf. the Matern hard core process (Matérn (1986, p. 37))). Now, for such a $W$, dependence neighbourhoods of $X_{\alpha}$ are often more naturally expressed geometrically, as subsets of $\Gamma$ (in the example above, we would take $N\left(C_{\alpha}\right)=B(\alpha, 10 \rho)$ ), and the number, $H\left(N\left(C_{\alpha}\right)\right)$, of random variables $F_{\gamma}$ with indices in $N\left(C_{\alpha}\right)$ is random and, in principle, unbounded, implying that $\kappa=\infty$. Furthermore, to match the setting of Chen and Shao (2004), the random variables $F_{\alpha}$ would need to be centred. However, it is often more natural to take arbitrary $F_{\alpha}$ and to centre $W$ by its expectation, $\int_{\Gamma} \mathrm{E}\left\{F_{\alpha} H(\mathrm{~d} \alpha)\right\}$, thus fully incorporating into $W$ the randomness arising as a result of the random number of summands. Although these differences can in principle be circumvented by special arguments in particular applications - such as, for example, by discretization and the introduction of a dependency graph, as in Penrose and Yukich (2005) - it is tedious to have to do so and the essential argument becomes obscured. In contrast, our Corollary 2.2 furnishes an analogue of Theorem 2.4 of Chen and Shao (2004) which is easy to apply and gives good results.

Our setting is described and the main theorems stated in Section 2. As far as possible, to facilitate comparison, we follow the presentation of Chen and Shao (2004). In Section 3, we give three applications, one from insurance mathematics and two from geometrical probability, exhibiting some improvement over previously known results. The proofs of the main theorems are given in Section 4.

\section{Main theorems}

Let $\Gamma$ be a locally compact, second-countable Hausdorff topological space with separable and complete metric $d$ (Kallenberg (1983, p. 11)) and Borel $\sigma$-field $\mathscr{B}(\Gamma)$, and let $\mathscr{H}$ denote the space of all finite, nonnegative, integer-valued measures on $\Gamma$ with $\sigma$-field $\mathscr{B}(\mathscr{H})$ generated by the 
weak topology. ( $\xi_{n} \in \mathscr{H}$ tends to $\xi$ in the weak topology on $\mathscr{H}$ if and only if $\int_{\Gamma} f \mathrm{~d} \xi_{n} \rightarrow \int_{\Gamma} f \mathrm{~d} \xi$ for all bounded, continuous functions $f$ on $\Gamma$ (Kallenberg (1983, p. 169)).) Throughout the section, we assume that $X=\left\{X_{\alpha}, \alpha \in \Gamma\right\}$ is a random field on $\Gamma$ and that $H$ is a point process on $\Gamma$ with locally finite mean measure $\mu$; that is,

$$
X:(\Gamma \times \Omega, \mathcal{B}(\Gamma) \times \mathcal{F}) \rightarrow(\mathbb{R}, \mathscr{B}(\mathbb{R})) \quad \text { and } \quad H:(\Omega, \mathcal{F}) \rightarrow(\mathscr{H}, \mathscr{B}(\mathcal{H}))
$$

are measurable mappings from an underlying probability space $(\Omega, \mathcal{F}, \mathrm{P})$. We also define $\mathcal{X}$ to be the space of all signed measures $v$ such that $v^{+}$and $v^{-}$are finite measures on $\Gamma$, and use $\mathscr{B}(\mathcal{X})$ to stand for the $\sigma$-field generated by the weak topology. For each set $B \in \mathscr{B}(\Gamma)$, we use $\left.\xi\right|_{B}$ to stand for the signed measure of $\xi$ restricted to $B$; that is,

$$
\left.\xi\right|_{B}(C)=\xi(B \cap C) \quad \text { for all } C \in \mathcal{B}(\Gamma) .
$$

We say that $\left\{D_{\alpha}, \alpha \in \Gamma\right\}$ is a measurable system of neighbourhoods if, for each $\alpha \in \Gamma$, $D_{\alpha} \in \mathscr{B}(\Gamma)$ is a closed set containing $\alpha$ and the mapping $(\alpha, \xi, x) \mapsto\left(\alpha,\left.\xi\right|_{D_{\alpha}}, x\right)$ is a measurable mapping from $(\Gamma \times \mathcal{X} \times \mathbb{R}, \mathscr{B}(\Gamma) \times \mathscr{B}(\mathcal{X}) \times \mathscr{B}(\mathbb{R}))$ into itself. A sufficient condition for the measurability condition to hold is that $D=\left\{(\alpha, \beta): \beta \in D_{\alpha}, \alpha \in \Gamma\right\}$ is a measurable subset of the product space $\Gamma^{2}:=\Gamma \times \Gamma$ (Chen and Xia (2004)).

Let $\left\{N_{\alpha}, \alpha \in \Gamma\right\}$ be a measurable system of neighbourhoods and $f$ a measurable function on $\Gamma \times \mathcal{X} \times \mathbb{R}$ such that $\left\{F_{\alpha}:=f\left(\alpha,\left.H_{1}\right|_{N_{\alpha}}, X_{\alpha}\right), \alpha \in \Gamma\right\}$ is a random field satisfying

$$
\mathrm{E}\left[\int_{\Gamma}\left|f\left(\alpha,\left.H_{1}\right|_{N_{\alpha}}, X_{\alpha}\right)\right| H(\mathrm{~d} \alpha)\right]^{2}<\infty,
$$

where $H_{1}(\mathrm{~d} \beta):=X_{\beta} H(\mathrm{~d} \beta)$. Our main object of interest is the random variable

$$
W:=\int_{\Gamma} F_{\alpha} H(\mathrm{~d} \alpha),
$$

the measurability of which can be proved by first considering $F_{\alpha}$ which are indicator functions of rectangular sets in $\mathscr{B}(\Gamma) \times \mathcal{F}$ and then extending to general random fields using the usual measure-theoretic techniques.

We now write $H_{2}(\mathrm{~d} \beta):=F_{\beta} H(\mathrm{~d} \beta)$, so that $W$ can be expressed as $H_{2}(\Gamma)$, and define the mean (signed) measure of $H_{2}$ by $\mu_{2}(A)=\mathrm{E} \int_{A} F_{\alpha} H(\mathrm{~d} \alpha)$, for a generic set $A$. It is a standard exercise to show that $\mu_{2}$ is absolutely continuous with respect to $\mu$; hence, we can define

$$
\bar{F}_{\alpha}=\frac{\mathrm{d} \mu_{2}}{\mathrm{~d} \mu}(\alpha), \quad \mu \text {-almost surely }
$$

(Kallenberg (1983, pp. 83-84)). When $H$ is a simple point process (Kallenberg (1983, p. 5)), $\bar{F}_{\alpha}$ can be intuitively interpreted as the conditional expectation of $F_{\alpha}$ given that there is a point of $H$ at $\alpha$. It then follows from the definition of $\bar{F}$ that $\mu_{2}(\mathrm{~d} \beta)=\bar{F}_{\beta} \mu(\mathrm{d} \beta)$. Now, for later use, define

$$
\begin{gathered}
\vartheta^{2}:=\operatorname{var} W, \quad G(\mathrm{~d} \alpha):=\left|F_{\alpha}\right| H(\mathrm{~d} \alpha)+\left|\bar{F}_{\alpha}\right| \mu(\mathrm{d} \alpha), \\
\tilde{H}_{2}(\mathrm{~d} \alpha):=\vartheta^{-1}\left[F_{\alpha} H(\mathrm{~d} \alpha)-\bar{F}_{\alpha} \mu(\mathrm{d} \alpha)\right] .
\end{gathered}
$$

Thus, the standardized version $\tilde{W}:=\vartheta^{-1}(W-\mathrm{E} W)$ can be expressed as $\tilde{H}_{2}(\Gamma)$. Finally, note that if we take $\Gamma=\{1,2, \ldots, n\}, H(\mathrm{~d} \alpha)=\delta_{\alpha}$, and $F_{\alpha}=X_{\alpha}-\mathrm{E} X_{\alpha}$, then we recover the setting of Chen and Shao (2004). 
Our interest is in studying normal approximation to the distribution $\mathcal{L}(W)$ of $W$ under various assumptions of local dependence, parallel to those in Chen and Shao (2004). With $B(\alpha, r)=\{y: d(y, \alpha) \leq r\}$, these can be expressed as follows.

(LD1) There exist a sequence, $r_{n} \downarrow 0$, and a measurable system of neighbourhoods, $\left\{A_{\alpha, n}\right.$, $\alpha \in \Gamma\}$, such that

(a) $A_{\alpha, n} \downarrow A_{\alpha}$ and $\left.H_{2}\right|_{B\left(\alpha, r_{n}\right)}$ is independent of $\left.H_{2}\right|_{A_{\alpha, n}^{\mathrm{c}}}$;

(b) if $B\left(\alpha, r_{n}\right) \subset B\left(\beta, r_{m}\right)$ then $A_{\alpha, n} \subset A_{\beta, m}$.

(LD2) Condition (LD1) holds and

(c) there exists a measurable system of neighbourhoods, $\left\{B_{\alpha}, \alpha \in \Gamma\right\}$, such that, for each $\alpha \in \Gamma, B_{\alpha} \supset A_{\alpha}$ and $\left.H_{2}\right|_{A_{\alpha}}$ is independent of $\left.H_{2}\right|_{B_{\alpha}^{\mathrm{c}}}$.

(LD3) Condition (LD2) holds and

(d) there exists a measurable system of neighbourhoods, $\left\{C_{\alpha}, \alpha \in \Gamma\right\}$, such that, for each $\alpha \in \Gamma, C_{\alpha} \supset B_{\alpha}$ and $\left.H_{2}\right|_{B_{\alpha}}$ is independent of $\left.H_{2}\right|_{C_{\alpha}^{c}}$.

Remark 2.1. Local dependence can also be defined in terms of Palm distributions, as in Chen and Xia (2004), resulting in the same condition as (LD1).

To state the theorems, we also define the following notation:

$$
Y_{\alpha}:=\int_{A_{\alpha}} \tilde{H}_{2}(\mathrm{~d} \beta)=\tilde{H}_{2}\left(A_{\alpha}\right), \quad Z_{\alpha}:=\tilde{H}_{2}\left(B_{\alpha}\right), \quad U_{\alpha}:=\tilde{H}_{2}\left(C_{\alpha}\right) .
$$

We write $\left|\tilde{H}_{2}\right|(A)=\int_{A}\left|\tilde{H}_{2}(\mathrm{~d} \alpha)\right|$ for a generic set $A$, and set

$$
\hat{K}(t, \mathrm{~d} \alpha)=\left\{\mathbf{1}_{\left\{-Y_{\alpha} \leq t<0\right\}}-\mathbf{1}_{\left\{0 \leq t \leq-Y_{\alpha}\right\}}\right\} \tilde{H}_{2}(\mathrm{~d} \alpha), \quad \hat{K}(t)=\int_{\Gamma} \hat{K}(t, \mathrm{~d} \alpha), \quad K(t)=\mathrm{E} \hat{K}(t),
$$

where $\mathbf{1}_{\{\cdot\}}$ is the indicator function. We then define the set

$$
B^{*}:=\left\{(\alpha, \beta): A_{\alpha} \cap B_{\beta} \neq \varnothing \text { and } B_{\alpha} \cap A_{\beta} \neq \varnothing\right\}
$$

thus, $Y_{\alpha}$ and $Y_{\beta}$ are independent if $(\alpha, \beta) \notin B^{*}$. Finally, for any $B \subset \Gamma$ we define

$$
N(B):=\left\{\beta \in \Gamma: B_{\beta} \cap B \neq \varnothing\right\} .
$$

Throughout the paper, we use $\tilde{H}_{2}^{*}$ to stand for an independent copy of $\tilde{H}_{2}$, and $Y_{\alpha}^{*}, Z_{\alpha}^{*}$, and $G^{*}$ are defined in terms of $\tilde{H}_{2}^{*}$ in the same way that $Y_{\alpha}, Z_{\alpha}$, and $G$ are defined in terms of $\tilde{H}_{2}$.

Our first theorem is then a rather direct counterpart to Theorem 2.1 of Chen and Shao (2004).

Theorem 2.1. Under condition (LD1), we have

$$
d_{\mathrm{K}}\left(\mathcal{L}\left(\vartheta^{-1}(W-\mathrm{E} W)\right), \mathcal{N}(0,1)\right) \leq r_{1}+4 r_{2}+8 r_{3}+r_{4}+4.5 r_{5}+1.5 r_{6},
$$


where

$$
\begin{gathered}
r_{1}=\mathrm{E}\left|\int_{\Gamma} Y_{\alpha} \tilde{H}_{2}(\mathrm{~d} \alpha)-\mathrm{E} \int_{\Gamma} Y_{\alpha} \tilde{H}_{2}(\mathrm{~d} \alpha)\right|, \quad r_{2}=\mathrm{E} \int_{\Gamma}\left|Y_{\alpha}\right| \mathbf{1}_{\left\{\left|Y_{\alpha}\right| \geq 1\right\}}\left|\tilde{H}_{2}(\mathrm{~d} \alpha)\right|, \\
r_{3}=\mathrm{E} \int_{\Gamma}\left\{Y_{\alpha}^{2} \wedge 1\right\}\left|\tilde{H}_{2}(\mathrm{~d} \alpha)\right|, \quad r_{4}=\mathrm{E}\left\{\left|\tilde{H}_{2}(\Gamma)\right| \int_{\Gamma}\left\{Y_{\alpha}^{2} \wedge 1\right\}\left|\tilde{H}_{2}(\mathrm{~d} \alpha)\right|\right\} \\
r_{5}=\int_{|t| \leq 1} \operatorname{var}(\hat{K}(t)) \mathrm{d} t \\
=\mathrm{E}\left\{\iint_{\Gamma^{2}} \tilde{H}_{2}(\mathrm{~d} \alpha) \tilde{H}_{2}(\mathrm{~d} \beta) \mathbf{1}_{\left\{Y_{\alpha} Y_{\beta}>0\right\}}\left(\left|Y_{\alpha}\right| \wedge\left|Y_{\beta}\right| \wedge 1\right)\right. \\
\left.\quad-\iint_{\Gamma^{2}} \tilde{H}_{2}(\mathrm{~d} \alpha) \tilde{H}_{2}^{*}(\mathrm{~d} \beta) \mathbf{1}_{\left\{Y_{\alpha} Y_{\beta}^{*}>0\right\}}\left(\left|Y_{\alpha}\right| \wedge\left|Y_{\beta}^{*}\right| \wedge 1\right)\right\} \\
r_{6}^{2}=\int_{|t| \leq 1}|t| \operatorname{var}\left(\hat{K}_{(t)) \mathrm{d} t}\right. \\
=\frac{1}{2} \mathrm{E}\left\{\iint_{\Gamma^{2}} \tilde{H}_{2}(\mathrm{~d} \alpha) \tilde{H}_{2}(\mathrm{~d} \beta) \mathbf{1}_{\left\{Y_{\alpha} Y_{\beta}>0\right\}}\left(Y_{\alpha}^{2} \wedge Y_{\beta}^{2} \wedge 1\right)\right. \\
\left.\quad-\iint_{\Gamma^{2}} \tilde{H}_{2}(\mathrm{~d} \alpha) \tilde{H}_{2}^{*}(\mathrm{~d} \beta) \mathbf{1}_{\left\{Y_{\alpha} Y_{\beta}^{*}>0\right\}}\left(Y_{\alpha}^{2} \wedge Y_{\beta}^{* 2} \wedge 1\right)\right\} .
\end{gathered}
$$

Our second theorem differs from its counterpart in Chen and Shao (2004), because the sums $\sum_{i=1}^{n}\left|Y_{i}\right|^{q}$, for $q=p$ and $q=p_{3}:=\min \{p, 3\}$, appearing there do not seem natural in our context. Instead, we prove the following variant.

Theorem 2.2. If condition (LD2) holds and $2<p \leq 4$, then

$$
d_{\mathrm{K}}\left(\mathcal{L}\left(\vartheta^{-1}(W-\mathrm{E} W)\right), \mathcal{N}(0,1)\right) \leq 15 \tilde{r}_{1}\left(p_{3}\right)+\frac{11}{2} \tilde{r}_{2}\left(p_{3}\right)+\left(1+\frac{3 \sqrt{2}}{4}\right) \sqrt{\tilde{r}_{2}(p)},
$$

where $p_{3}:=\min \{p, 3\}$ and

$$
\begin{aligned}
\tilde{r}_{1}(q) & :=\mathrm{E} \int_{\Gamma}\left|Y_{\alpha}\right|^{q-1}\left|\tilde{H}_{2}(\mathrm{~d} \alpha)\right| \leq \frac{1}{\vartheta q} \mathrm{E} \int_{\Gamma} G\left(A_{\alpha}\right)^{q-1} G(\mathrm{~d} \alpha), \\
\tilde{r}_{2}(q) & :=\mathrm{E} \iint_{B^{*}}\left|Y_{\alpha}\right|^{q-2}\left|\tilde{H}_{2}(\mathrm{~d} \alpha)\right|\left\{\left|\tilde{H}_{2}(\mathrm{~d} \beta)\right|+\left|\tilde{H}_{2}^{*}(\mathrm{~d} \beta)\right|\right\} \\
& \leq \frac{1}{\vartheta^{q}} \mathrm{E} \int_{\Gamma} G\left(A_{\alpha}\right)^{q-2}\left[G\left(N\left(A_{\alpha}\right)\right)+G^{*}\left(N\left(A_{\alpha}\right)\right)\right] G(\mathrm{~d} \alpha) .
\end{aligned}
$$

The next theorem also differs a little from its counterpart, Theorem 2.3, in Chen and Shao (2004). Their error terms $r_{7}$ and $r_{11}$ have disappeared from the upper bound at the cost of some minor modification of $r_{8}$ and $r_{9}$. The term $r_{8}^{\prime}$ is needed because our setting is more general than theirs. The other extra terms appear because our concentration inequality in Proposition 4.1 is slightly different; we were unable to reproduce their proof in full detail.

Theorem 2.3. Suppose that condition (LD3) is satisfied. Then

$$
\begin{aligned}
& d_{\mathrm{K}}\left(\mathcal{L}\left(\vartheta^{-1}(W-\mathrm{E} W)\right), \mathcal{N}(0,1)\right) \\
& \quad \leq 4 r_{2}+\left(3+r_{13}\right) r_{3}+\left(2.1+\frac{1}{2} r_{13}\right) r_{8}+\left(1.1+\frac{1}{2} r_{13}\right) r_{8}^{\prime}+r_{9}+2 r_{10}+r_{12}+r_{14}
\end{aligned}
$$


and

$$
d_{\mathrm{K}}\left(\mathcal{L}\left(\vartheta^{-1}(W-\mathrm{E} W)\right), \mathcal{N}(0,1)\right) \leq 4 r_{2}+4 r_{3}+3 r_{8}+2 r_{8}^{\prime}+r_{9}+2 r_{10}+r_{12}+r_{13},
$$

where

$$
\begin{gathered}
r_{8}=\mathrm{E} \int_{\Gamma}\left(\left|Y_{\alpha}\right| \wedge 1\right)\left|Z_{\alpha}\right|\left|\tilde{H}_{2}(\mathrm{~d} \alpha)\right|, \quad r_{8}^{\prime}=\mathrm{E} \int_{\Gamma}\left|Z_{\alpha}\right|\left(\left|Y_{\alpha}^{*}\right| \wedge 1\right)\left|\tilde{H}_{2}^{*}(\mathrm{~d} \alpha)\right|, \\
r_{9}=\mathrm{E} \int_{\Gamma}\left|\tilde{H}_{2}(\Gamma)\right|\left(\left|Z_{\alpha}\right| \wedge 1\right)\left(\left|Y_{\alpha}\right| \wedge 1\right)\left|\tilde{H}_{2}(\mathrm{~d} \alpha)\right| \\
r_{10}=\mathrm{E} \iint_{B^{*}}\left\{\left(\left|Y_{\beta_{1}}\right| \wedge\left|Y_{\beta_{2}}\right| \wedge 1\right)\left|\tilde{H}_{2}\left(\mathrm{~d} \beta_{1}\right)\right|\left|\tilde{H}_{2}\left(\mathrm{~d} \beta_{2}\right)\right|\right. \\
\left.\quad+\left(\left|Y_{\beta_{1}}\right| \wedge\left|Y_{\beta_{2}}^{*}\right| \wedge 1\right)\left|\tilde{H}_{2}\left(\mathrm{~d} \beta_{1}\right)\right|\left|\tilde{H}_{2}^{*}\left(\mathrm{~d} \beta_{2}\right)\right|\right\} \\
r_{12}=\mathrm{E} \int_{\Gamma}\left(\left|\tilde{H}_{2}(\Gamma)\right|+1\right)\left(\left|Z_{\alpha}\right| \wedge 1\right)\left(\left|Y_{\alpha}^{*}\right| \wedge 1\right)\left|\tilde{H}_{2}^{*}(\mathrm{~d} \alpha)\right| \\
r_{13}=\sup _{\alpha \in \Gamma} \mathrm{E} \int_{N\left(C_{\alpha}\right)}\left|\tilde{H}_{2}(\mathrm{~d} \beta)\right|, \\
r_{14}=\sup _{\alpha \in \Gamma} \mathrm{E} \int_{N\left(C_{\alpha}\right)}\left(\left|Y_{\beta}\right| \wedge 1\right)\left|\tilde{H}_{2}(\mathrm{~d} \beta)\right| .
\end{gathered}
$$

The statement of the next theorem is agreeably compact.

Theorem 2.4. Suppose that condition (LD3) is satisfied and that $2<p \leq 3$. Then

$$
d_{\mathrm{K}}\left(\mathcal{L}\left(\vartheta^{-1}(W-\mathrm{E} W)\right), \mathcal{N}(0,1)\right) \leq 16 \eta_{1}+8 \eta_{2}+\vartheta^{-1} \sup _{\alpha} \mathrm{E} G\left(N\left(C_{\alpha}\right)\right),
$$

where

$$
\begin{aligned}
& \eta_{1}:=\vartheta^{-p} \mathrm{E} \int_{\Gamma} G\left(N\left(C_{\alpha}\right)\right)^{p-1} G(\mathrm{~d} \alpha), \\
& \eta_{2}:=\vartheta^{-p} \mathrm{E} \int_{\Gamma} G\left(N\left(C_{\alpha}\right)\right)^{p-2} G^{*}\left(N\left(C_{\alpha}\right)\right) G(\mathrm{~d} \alpha) .
\end{aligned}
$$

Now let $R(\mathrm{~d} \alpha):=\left|F_{\alpha}\right| H(\mathrm{~d} \alpha)$, whence $G(\mathrm{~d} \alpha) \leq R(\mathrm{~d} \alpha)+\mathrm{E} R(\mathrm{~d} \alpha)$; in practice $R$ is usually the easiest quantity to work with. Define the following measures of smallness:

$$
\begin{aligned}
\varepsilon_{1}(q) & :=\vartheta^{-q} \mathrm{E} \int_{\alpha \in \Gamma} R\left(N\left(A_{\alpha}\right)\right)^{q-1} R(\mathrm{~d} \alpha), \\
\varepsilon_{2}(q) & :=\vartheta^{-q} \int_{\alpha \in \Gamma} \mathrm{E} R\left(N\left(A_{\alpha}\right)\right)^{q-1} \mathrm{E} R(\mathrm{~d} \alpha), \\
\varepsilon_{3} & :=\vartheta^{-p} \mathrm{E} \int_{\alpha \in \Gamma} R\left(N\left(C_{\alpha}\right)\right)^{p-1} R(\mathrm{~d} \alpha), \\
\varepsilon_{4} & :=\vartheta^{-p} \int_{\alpha \in \Gamma} \mathrm{E} R\left(N\left(C_{\alpha}\right)\right)^{p-1} \mathrm{E} R(\mathrm{~d} \alpha), \\
\varepsilon_{5} & :=\vartheta^{-1} \sup _{\alpha \in \Gamma} \mathrm{E} R\left(N\left(C_{\alpha}\right)\right) .
\end{aligned}
$$

We can then bound the errors in Theorems 2.2 and 2.4 in terms of these quantities. It follows, after some calculation, that, for $q \geq 2$,

$$
\tilde{r}_{1}(q) \leq 2^{q-2}\left\{\varepsilon_{1}(q)+3 \varepsilon_{2}(q)\right\}, \quad \tilde{r}_{2}(q) \leq 6 \times 2^{(q-3)+}\left\{\varepsilon_{1}(q)+3 \varepsilon_{2}(q)\right\},
$$


and that, for $2 \leq p \leq 3$,

$$
\eta_{1} \leq 2\left(\varepsilon_{3}+3 \varepsilon_{4}\right), \quad \eta_{2} \leq 2\left(\varepsilon_{3}+4 \varepsilon_{4}\right)
$$

This leads to the following corollaries.

Corollary 2.1. Under the conditions of Theorem 2.2 , for $2<p \leq 4$ and with $p_{3}:=\min \{p, 3\}$ we have

$$
d_{\mathrm{K}}\left(\mathcal{L}\left(\vartheta^{-1}(W-\mathrm{E} W)\right), \mathcal{N}(0,1)\right) \leq 63\left\{\varepsilon_{1}\left(p_{3}\right)+3 \varepsilon_{2}\left(p_{3}\right)\right\}+8 \sqrt{\varepsilon_{1}(p)+3 \varepsilon_{2}(p)} .
$$

Corollary 2.2. Under the conditions of Theorem 2.4 , for $2<p \leq 3$ we have

$$
d_{\mathrm{K}}\left(\mathcal{L}\left(\vartheta^{-1}(W-\mathrm{E} W)\right), \mathcal{N}(0,1)\right) \leq 48 \varepsilon_{3}+160 \varepsilon_{4}+2 \varepsilon_{5} .
$$

\section{Applications}

\subsection{An insurance model}

A simple model in insurance assumes that each of a large number of insured risks has a small probability of resulting in a claim, independently of the others, and that the claim amounts are independent and identically distributed random variables which are also independent of the number of claims. Hence, the total number of claims approximately follows a Poisson distribution, leading to a compound Poisson model for the total amount of the claims. Goovaerts and Dhaene (1996) showed that a compound Poisson distribution is still a valid approximation for the total claim amount, even if the occurrences of the claims are weakly dependent, as long as the claim amounts are still independent and identically distributed random variables which are also independent of the number of claims.

When the time scale is taken into consideration, the total sum of the claims on an insurance portfolio is classically modelled as

$$
S(t) \equiv S_{N(t)}=\left\{\begin{array}{ll}
0, & N(t)=0, \\
\xi_{1}+\cdots+\xi_{N(t)}, & N(t) \geq 1,
\end{array} \quad t \geq 0,\right.
$$

where $\left\{\xi_{i}, i \geq 1\right\}$ are independent and identically distributed random variables representing the amounts of the claims and the claim number process $\{N(t), t \geq 0\}$, which records the numbers and times of the insurance claims, is a counting process independent of $\left\{\xi_{i}, i \geq 1\right\}$ (Embrechts et al. (1997, pp. 96-111)). When $\{N(t), t \geq 0\}$ is a renewal process, the process $\{S(t), t \geq 0\}$ is the well-known Cramér-Lundberg model (Embrechts et al. (1997, p. 22)). While this model has been extensively studied and used, it may seem unnatural to assume that the claim sizes are independent and identically distributed, or that the claims occur in a renewal process; natural disasters, for instance, could induce local temporal dependence in both the sizes and the numbers of claims. There have been numerous attempts to address the issue as regards the claim number process, by assuming it variously to be a stationary point process, a process with independent increments, a mixed Poisson process, a negative binomial process, or a pure-birth Markov process (see Rolski et al. (1999) or Embrechts et al. (1997) for details), but relatively little work addresses the interdependence of claim sizes.

In what follows, we let $\left\{Y_{t}, t \geq 0\right\}$ be a strictly stationary process representing a random process describing the claim environment over time, and let $H$ be a simple point process on 
$\Gamma:=[0, T] \times \mathbb{N}$ recording the times and sizes of clusters of claims. We do not necessarily require that

$$
H(\mathrm{~d} s, \mathbb{N}):=\sum_{n \geq 1} H(\mathrm{~d} s, n)
$$

should be absolutely continuous with respect to Lebesgue measure, as this facilitates application to daily aggregated data. If $H\{\alpha\}=1$ for $\alpha=(t, n)$ then the total claim amount $X_{\alpha}$ is assumed, conditionally on the value, $y$, of $Y_{t}$, to be a sum of $n$ independent, identically distributed random variables $Z_{i}^{(t)}$ with distribution $Q^{(y)}$, depending only on $y$, having mean $m_{1}(y)$, variance $v(y)$, and finite third absolute moment $m_{3}^{3}(y)$. We also write

$$
\bar{m}_{3}^{3}(y):=\mathrm{E}\left\{\left|Z_{1}^{(0)}-\mathrm{E} m_{1}\left(Y_{0}\right)\right|^{3} \mid Y_{0}=y\right\}
$$

and write $\tilde{X}_{\alpha}$ for the precentred claim amount $X_{\alpha}-n \mathrm{E} m_{1}\left(Y_{0}\right)$.

In order to have only local dependence, we assume that $\left\{Y_{t}, t \geq 0\right\}$ is independent of $H$ and that there exists an $h_{0}>0$ such that, for all $a$ and $b, 0<a<b<\infty,\left.Y\right|_{[a, b]}$ is independent of $\left.Y\right|_{\mathbb{R} \backslash\left(a-h_{0}, b+h_{0}\right)}$ and $\left.H\right|_{[a, b] \times \mathbb{N}}$ is independent of $\left.H\right|_{\left(\mathbb{R} \backslash\left(a-h_{0}, b+h_{0}\right)\right) \times \mathbb{N}}$. Then, in order to obtain explicit bounds, we assume that there exist a positive constant $\beta$, probabilities $\left\{p_{j}, j \geq 1\right\}$, and a measure, $\mu^{*}$, on $(0, T]$ such that, for $\alpha_{i}=\left(t_{i}, n_{i}\right), 1 \leq i \leq 3$,

$$
\begin{aligned}
\mathrm{E} H\left(\mathrm{~d} \alpha_{1}\right) & \leq p_{n_{1}} \mu^{*}\left(\mathrm{~d} t_{1}\right), \\
\mathrm{E}\left\{H\left(\mathrm{~d} \alpha_{1}\right) H\left(\mathrm{~d} \alpha_{2}\right)\right\} & \leq \beta p_{n_{1}} p_{n_{2}} \mu^{*}\left(\mathrm{~d} t_{1}\right) \mu^{*}\left(\mathrm{~d} t_{2}\right) \quad \\
\mathrm{E}\left\{H\left(\mathrm{~d} \alpha_{1}\right) H\left(\mathrm{~d} \alpha_{2}\right) H\left(\mathrm{~d} \alpha_{3}\right)\right\} & \leq \beta^{2} p_{n_{1}} p_{n_{2}} p_{n_{3}} \mu^{*}\left(\mathrm{~d} t_{1}\right) \mu^{*}\left(\mathrm{~d} t_{2}\right) \mu^{*}\left(\mathrm{~d} t_{3}\right) \\
& \text { if } t_{1}, t_{2}, \text { and } t_{3} \text { are distinct, }
\end{aligned}
$$

Thus, $\mu^{*}(\mathrm{~d} s) \geq \mathrm{E} H(\mathrm{~d} s, \mathbb{N})$ can be thought of as determining a typical maximal rate of occurrence of clusters of claims, the $p_{j}$ as controlling the sizes of the clusters, and $\beta$ as a factor reflecting the extra intensity of clusters of claims at time $t$, if it is known that a cluster has already occurred within the interval $\left[t-h_{0}, t+h_{0}\right]$. We shall further assume that $\mu^{*}(s, s+h] \leq \mu_{+} h$ for some $\mu_{+}<\infty$, whenever $h \geq h_{0}$. We also define

$$
\begin{gathered}
m_{3}^{3}:=\operatorname{E} m_{3}^{3}\left(Y_{0}\right), \quad \bar{m}_{3}^{3}:=\operatorname{E~}_{3}^{3}\left(Y_{0}\right), \quad n_{+}^{3}:=\sum_{n \geq 1} n^{3} p_{n}, \\
\bar{\mu}:=T^{-1} \int_{0}^{T} \operatorname{E} H(\mathrm{~d} t, \mathbb{N}), \quad \bar{n}^{3}:=T^{-1} \int_{0}^{T} \sum_{n \geq 1} n^{3} \operatorname{E} H(\mathrm{~d} t, n) .
\end{gathered}
$$

Here $m_{3}$ and $\bar{m}_{3}$ are respectively generous measures of the typical individual claim size and its deviation from its mean, and $\bar{\mu}$ and $\bar{n}$ are respectively measures of the typical rate of occurrence and size of a cluster of claims. To make our estimates of approximation error useful, we assume that all of these quantities are finite.

We investigate normal approximations to two versions of the total claim amount in the interval $[0, T]$ considered previously in the literature: the natural version, $W:=\int_{\Gamma} X_{\alpha} H(\mathrm{~d} \alpha)$, and the precentred version $W_{0}:=\int_{\Gamma} \tilde{X}_{\alpha} H(\mathrm{~d} \alpha)$. For each of these, an assumption is needed to ensure that its variance is genuinely of asymptotic order $T$ as $T$ increases. If, for each 
$s \in[0, T]$, the inequality

$$
\begin{aligned}
& \int_{\left(s-h_{0}\right)_{+}}^{\left(s+h_{0}\right) \wedge T} \mathbf{1}_{\{t \neq s\}} \sum_{n, r \geq 1} n r\left\{\mathrm{E}\left\{m_{1}\left(Y_{t}\right) m_{1}\left(Y_{s}\right)\right\} \mathrm{E}\{H(\mathrm{~d} s, n) H(\mathrm{~d} t, r)\}\right. \\
& \left.\quad-\left(\mathrm{E} m_{1}\left(Y_{0}\right)\right)^{2} \mathrm{E} H(\mathrm{~d} s, n) \mathrm{E} H(\mathrm{~d} t, r)\right\} \\
& +\sum_{n \geq 1}\left\{n^{2} \mathrm{E} m_{1}\left(Y_{0}\right)^{2}+n \mathrm{E} v\left(Y_{0}\right)\right\} \mathrm{E} H(\mathrm{~d} s, n) \\
& -\left(\mathrm{E} m_{1}\left(Y_{0}\right)\right)^{2}\left\{\sum_{n \geq 1} n \mathrm{E} H(\mathrm{~d} s, n)\right\}^{2} \\
& \geq m_{3}^{2} \bar{n}^{2} \delta_{1} \mathrm{E} H(\mathrm{~d} s, \mathbb{N}),
\end{aligned}
$$

where $\int_{a}^{b}$ is to be interpreted as $\int_{(a, b]}$, holds for some $\delta_{1}>0$, then

$$
\vartheta^{2}:=\operatorname{var} W \geq T \bar{\mu} m_{3}^{2} \bar{n}^{2} \delta_{1} ;
$$

see (3.8), below. Similarly, if

$$
\begin{aligned}
& \int_{\left(s-h_{0}\right)_{+}}^{\left(s+h_{0}\right) \wedge T} \mathbf{1}_{\{t \neq s\}} \sum_{n \geq 1} \sum_{r \geq 1} n r\left[\mathrm{E}\left\{m_{1}\left(Y_{t}\right) m_{1}\left(Y_{s}\right)\right\}-\left(\mathrm{E} m_{1}\left(Y_{0}\right)\right)^{2}\right] \mathrm{E}\{H(\mathrm{~d} s, n) H(\mathrm{~d} t, r)\} \\
& +\sum_{n \geq 1}\left\{n^{2} \operatorname{var} m_{1}\left(Y_{0}\right)+n \mathrm{E} v\left(Y_{0}\right)\right\} \mathrm{E} H(\mathrm{~d} s, n) \\
& \quad \geq \bar{m}_{3}^{2} \bar{n}^{2} \delta_{2} \mathrm{E} H(\mathrm{~d} s, \mathbb{N})
\end{aligned}
$$

holds for some $\delta_{2}>0$, then

$$
\vartheta_{0}^{2}:=\operatorname{var} W_{0} \geq T \bar{\mu} \bar{m}_{3}^{2} \bar{n}^{2} \delta_{2} .
$$

The quantities $\delta_{1}$ and $\delta_{2}$ are a rough measure of the factor by which the variance is altered in the two cases as a result of the presence of local dependence. If there were no local dependence in either the $Y$ or the $H$ process, and if $\mathrm{E} H(\mathrm{~d} s, n)=\bar{p}_{n} \bar{\mu} \mathrm{d} s$, in which case $H$ would be a Poisson cluster process, then the left-hand side of (3.4) would reduce to

$$
\left(\mathrm{E} N^{2} \mathrm{E} m_{1}\left(Y_{0}\right)^{2}+\mathrm{E} N \mathrm{E} v\left(Y_{0}\right)\right) \bar{\mu} \mathrm{d} s,
$$

where $N$ is a random variable with the cluster size distribution $\left\{\bar{p}_{j}, j \geq 1\right\}$. The factor $m_{3}^{2} \bar{n}^{2}$ on the right-hand side of (3.4) is chosen to mirror the corresponding contribution to (3.6), albeit in a somewhat simplified way. Now $\delta_{1}$ can be seen as a modification arising because of the dependence structure. The occurrence of dependent claims would in practice be expected to increase the variance, meaning that we would expect to have $\delta_{1}>1$, so the assumption that $\delta_{1}>0$ in (3.4) is reasonable. A similar interpretation can be made for $\delta_{2}$, appearing in (3.5). There, if all the claim size distributions were identical, meaning that the $Y$ process played no part, then the left-hand size of (3.5) would actually simplify further to $v \mathrm{E} N \bar{\mu} \mathrm{d} s$, where $v$ is the variance of the individual claim amounts.

Theorem 3.1. Under the assumptions in the preceding paragraphs, if (3.4) holds then

$$
d_{\mathrm{K}}\left(\mathcal{L}\left(\vartheta^{-1}(W-\mathrm{E} W)\right), \mathcal{N}(0,1)\right)=O\left(\{\bar{\mu} T\}^{-1 / 2}\right),
$$


and if (3.5) holds then

$$
d_{\mathrm{K}}\left(\mathcal{L}\left(\vartheta_{0}^{-1}\left(W_{0}-\mathrm{E} W_{0}\right)\right), \mathcal{N}(0,1)\right)=O\left(\{\bar{\mu} T\}^{-1 / 2}\right) .
$$

Explicit bounds for the order terms are given in (3.9), below.

Proof. We use Corollary 2.2 with $p=3$ to prove the claims, noting that, for $\alpha=(t, n)$, we can take $A_{\alpha}=U(t, 1), B_{\alpha}=U(t, 2), C_{\alpha}=U(t, 3)$, and $N\left(C_{\alpha}\right)=U(t, 5)$, where $U(t, r):=\left(\left(t-r h_{0}, t+r h_{0}\right) \cap[0, T]\right) \times \mathbb{N}$.

First of all, for $W$, we have $R(\mathrm{~d} \alpha)=X_{\alpha} H(\mathrm{~d} \alpha)$, meaning that, for $\alpha=(t, n)$,

$$
\mathrm{E} R(\mathrm{~d} \alpha)=n \mathrm{E} m_{1}\left(Y_{0}\right) \mathrm{E} H(\mathrm{~d} t, n)
$$

and, hence, from (3.1), that

$$
\begin{aligned}
\operatorname{EE} R\left(N\left(C_{\alpha}\right)\right) & =\int_{\left(t-5 h_{0}, t+5 h_{0}\right) \cap[0, T]} \sum_{n \geq 1} n \operatorname{E~} m_{1}\left(Y_{0}\right) \operatorname{E} H(\mathrm{~d} s, n) \\
& \leq 10 n_{+} m_{3} \mu_{+} h_{0},
\end{aligned}
$$

giving

$$
\varepsilon_{5} \leq 10 \mu_{+} h_{0} n_{+} m_{3} \vartheta^{-1} .
$$

To find $\varepsilon_{3}$, we use (3.1)-(3.3) to give, for $\alpha=(t, n)$,

$$
\begin{aligned}
& \mathrm{E} \iint_{\beta, \gamma \in N\left(C_{\alpha}\right)} R(\mathrm{~d} \beta) R(\mathrm{~d} \gamma) R(\mathrm{~d} \alpha) \\
& \leq \iint_{u, v \in U(t, 5)} \sum_{r, s \geq 1} \frac{r^{3}+s^{3}+n^{3}}{3} m_{3}^{3} p_{r} p_{s} p_{n} \beta^{2} \mu^{*}(\mathrm{~d} u) \mu^{*}(\mathrm{~d} v) \mu^{*}(\mathrm{~d} t) \\
& \quad+2 \int_{u \in U(t, 5)} \sum_{r \geq 1} \frac{r^{3}+2 n^{3}}{3} m_{3}^{3} p_{r} p_{n} \beta \mu^{*}(\mathrm{~d} u) \mu^{*}(\mathrm{~d} t) \\
& \quad+\int_{u \in U(t, 5)} \sum_{r \geq 1} \frac{2 r^{3}+n^{3}}{3} m_{3}^{3} p_{r} p_{n} \beta \mu^{*}(\mathrm{~d} u) \mu^{*}(\mathrm{~d} t)+n^{3} m_{3}^{3} p_{n} \beta \mu^{*}(\mathrm{~d} t) \\
& \leq m_{3}^{3} p_{n} \mu^{*}(\mathrm{~d} t)\left\{100\left(\beta \mu_{+} h_{0}\right)^{2} \frac{2 n_{+}^{3}+n^{3}}{3}+20 \beta \mu_{+} h_{0} \frac{n_{+}^{3}+2 n^{3}}{3}\right. \\
& \left.\quad+10 \beta \mu_{+} h_{0} \frac{2 n_{+}^{3}+n^{3}}{3}+n^{3}\right\} .
\end{aligned}
$$

It follows that

$$
\varepsilon_{3} \leq \vartheta^{-3} n_{+}^{3} m_{3}^{3} \mu_{+} T\left\{1+30 \beta \mu_{+} h_{0}+100\left(\beta \mu_{+} h_{0}\right)^{2}\right\} .
$$

Likewise, it follows from (3.7), (3.1), and (3.2) that, for $\alpha=(t, n)$,

$$
\mathrm{E} R(\mathrm{~d} \alpha) \leq n m_{3} p_{n} \mu^{*}(\mathrm{~d} t)
$$

and

$$
\mathrm{E} R\left(N\left(C_{\alpha}\right)\right)^{2} \leq n_{+}^{2} m_{3}^{2} \mu_{+} h_{0}\left\{10+100 \beta \mu_{+} h_{0}\right\}
$$


giving

$$
\varepsilon_{4} \leq 10 \vartheta^{-3} n_{+}^{3} m_{3}^{3} \mu_{+}^{2} h_{0} T\left\{1+10 \beta \mu_{+} h_{0}\right\}
$$

Finally, by (3.4),

$$
\begin{aligned}
\vartheta^{2}= & \int_{0}^{T} \int_{0}^{T} \mathbf{1}_{\{t \neq s\}} \sum_{n, r \geq 1} n r\left\{\mathrm{E}\left\{m_{1}\left(Y_{t}\right) m_{1}\left(Y_{s}\right)\right\} \mathrm{E}\{H(\mathrm{~d} s, n) H(\mathrm{~d} t, r)\}\right. \\
& \left.+\int_{0}^{T} \sum_{n \geq 1}\left\{n^{2} \mathrm{E} m_{1}\left(Y_{0}\right)^{2}+n \mathrm{E} m_{1}\left(Y_{0}\right)\right)^{2} \mathrm{E} H(\mathrm{~d} s, n) \mathrm{E} H(\mathrm{~d} t, r)\right\} \\
& -\left(\mathrm{E} m_{1}\left(Y_{0}\right)\right)^{2} \int_{0}^{T}\left\{\sum_{n \geq 1} n \mathrm{E} H(\mathrm{~d} s, n)\right\}^{2} \\
= & \int_{0}^{T} \int_{\left(s-h_{0}\right)_{+}}^{\left(s+h_{0}\right) \wedge T} \mathbf{1}_{\{t \neq s\}} \sum_{n, r \geq 1} n r\left\{\mathrm{E}\left\{m_{1}\left(Y_{t}\right) m_{1}\left(Y_{s}\right)\right\} \mathrm{E}\{H(\mathrm{~d} s, n) H(\mathrm{~d} t, r)\}\right. \\
& +\int_{0}^{T} \sum_{n \geq 1}\left\{n^{2} \mathrm{E}_{1}\left(Y_{0}\right)^{2}+n \mathrm{E} v\left(Y_{0}\right)\right\} \mathrm{E} H(\mathrm{~d} s, n) \\
& \left.-\left(\mathrm{E} m_{1}\left(Y_{0}\right)\right)^{2} \mathrm{E} H(\mathrm{~d} s, n) \mathrm{E} H(\mathrm{~d} t, r)\right\} \\
\geq & T \bar{\mu} m_{3}^{2} \bar{n}^{2} \delta_{1} .
\end{aligned}
$$

By applying Corollary 2.2, we thus obtain the bound

$$
\begin{aligned}
& d_{\mathrm{K}}\left(\mathcal{L}\left(\vartheta^{-1}(W-\mathrm{E} W)\right), \mathcal{N}(0,1)\right) \\
& \leq \frac{1}{\sqrt{\bar{\mu} \delta_{1} T}}\left\{\delta _ { 1 } ^ { - 1 } \frac { n _ { + } ^ { 3 } \mu _ { + } } { \overline { n } ^ { 3 } \overline { \mu } } \left(48\left\{1+30 \beta \mu_{+} h_{0}+100\left(\beta \mu_{+} h_{0}\right)^{2}\right\}\right.\right. \\
& \left.\left.\quad+1600 \mu_{+} h_{0}\left\{1+10 \beta \mu_{+} h_{0}\right\}\right)+20 \frac{n_{+}}{\bar{n}} \mu_{+} h_{0}\right\} .
\end{aligned}
$$

The proof of the second approximation follows along exactly the same lines; the bound is as in (3.9), but with $\delta_{1}$ replaced by $\delta_{2}$.

The error bound contains factors, $n_{+} / \bar{n}$ and $\mu_{+} / \bar{\mu}$, which reflect the variability permitted in the specification of the system. The other element of particular interest is the product $\mu_{+} h_{0}$, which indicates the result of the dependence over time; it measures the maximal expected number of clusters of claims arising during an interval of length $h_{0}$. The bounds are strongly influenced by its value, which should ideally be as small as possible. This makes it sensible in practice to formulate the claims process in such a way that this is so. One way of doing this would be to add further structure to the process, indexing claims not only according to time of occurrence, but also by location and type of claim; it may be plausible to suppose that claims arising at a certain geographical distance from one another are independent, or that claims relating to different kinds of risk arise independently of one another. In such a scenario, the analogue of $\mu_{+} h_{0}$ is a corresponding measure of the expected number of clusters of claims in 
a region of dependence, but, because of the extra stratification according to the source of the claim, this can be expected to be much smaller.

\subsection{Local dependence in geometric probability}

Avram and Bertsimas (1993) showed that many statistics arising in geometric probability are closely equivalent to sums of random variables whose dependence structure, when expressed in terms of a dependency graph, exhibits neighbourhoods of rather small cardinality. This enables central limit theorems formulated for just these situations, such as that of Baldi and Rinott (1989), to be applied. Penrose and Yukich (2005) combined their ideas with the general notion of a stabilizing functional and with the theorems of Chen and Shao (2004), obtaining very good rates of convergence for the central limit theorem in a wide range of problems of this kind. Their examples include the total edge length of the $k$-nearest-neighbour graph, the number of edges in the sphere-of-influence graph, and the independence number of the $r$-threshold graph, all based on the points of an underlying realization of a Poisson process in a bounded region of $\mathbb{R}^{d}$. Here, we show that our modification of Chen and Shao's theory, as it was designed to, allows us to bypass the construction of a dependency graph, resulting in an argument which flows more naturally. As a by-product, the rates of convergence that we obtain are slightly better than those of Penrose and Yukich.

We begin by describing the setting of Penrose and Yukich (2005). We take $H$ to be a marked Poisson process on $\Gamma=\Gamma_{1} \times \Gamma_{2}$, where $\Gamma_{1}$ is a compact subset of $\mathbb{R}^{d}$ and $\Gamma_{2}$ is a mark space, assumed to be locally compact, second-countable, and Hausdorff. The mean measure of $H$ takes the form $\lambda \nu$, where $v$ is a probability measure on $\Gamma$ and $\lambda$, the average number of points of $H$, is assumed to be large. The marginal, $v_{1}$, of $v$ on $\Gamma_{1}$ has a probability density bounded by $\kappa<\infty$. For each $\alpha=\left(\alpha_{1}, \alpha_{2}\right) \in \Gamma$, we denote the conditional distribution of $v$ on the mark space $\Gamma_{2}$ by $\nu_{2}\left(\cdot \mid \alpha_{1}\right)$.

The random variable of interest is expressed as $W:=\int_{\Gamma} F_{\alpha} H(\mathrm{~d} \alpha)$, where $F_{\alpha}:=f_{\alpha}(H)$ and the functions $f_{\alpha}: \mathcal{X} \rightarrow \mathbb{R}$ are stabilizing in the following sense. Defining the neighbourhoods

$$
D\left(\alpha_{1}, \rho\right):=\left\{\left(\beta_{1}, \beta_{2}\right) \in \Gamma:\left|\beta_{1}-\alpha_{1}\right| \leq \rho\right\}
$$

for any $\rho \geq 0$, we suppose that for each $\alpha$ there is a function $r_{\alpha}: \mathcal{X} \rightarrow \mathbb{R}_{+}$with the property that, for each $\rho \in \mathbb{R}_{+}$and $\chi \in \mathcal{X}$,

$$
\mathbf{1}_{[0, \rho]}\left(r_{\alpha}(\chi)\right)=\tilde{r}_{\alpha}\left(\rho,\left.\chi\right|_{D\left(\alpha_{1}, \rho\right)}\right)
$$

for some measurable function $\tilde{r}_{\alpha}$, and

$$
Q(\rho):=\sup _{\alpha \in \Gamma} \mathrm{P}\left(r_{\alpha}(H)>\lambda^{-1 / d} \rho\right) \rightarrow 0 \quad \text { as } \rho \rightarrow \infty .
$$

Then the function $f_{\alpha}$ is assumed to be such that

$$
f_{\alpha}(\chi)=f_{\alpha}\left(\left.\chi\right|_{D\left(\alpha_{1}, \rho\right)}\right) \quad \text { for all } \rho \geq r_{\alpha}(\chi) .
$$

Combining this with (3.10), the loose interpretation is that the value of $f_{\alpha}$ is determined only by the configuration of the relatively few points closest to $\alpha$.

Setting $F_{\alpha}(\rho):=F_{\alpha} \mathbf{1}_{\left\{r_{\alpha}(H) \leq \lambda^{-1 / d} \rho\right\}}$, it thus follows that

$$
W(\rho):=\int_{\Gamma} F_{\alpha}(\rho) H(\mathrm{~d} \alpha)
$$


satisfies

$$
\mathrm{P}(W \neq W(\rho)) \leq \lambda Q(\rho)
$$

and that $W(\rho)$ fulfils the local dependence condition (LD3) with

$$
\begin{aligned}
A_{\alpha} & :=D\left(\alpha_{1}, 2 \lambda^{-1 / d} \rho\right), & B_{\alpha} & :=D\left(\alpha_{1}, 4 \lambda^{-1 / d} \rho\right), \\
C_{\alpha} & :=D\left(\alpha_{1}, 6 \lambda^{-1 / d} \rho\right), & N\left(C_{\alpha}\right) & :=D\left(\alpha_{1}, 10 \lambda^{-1 / d} \rho\right) .
\end{aligned}
$$

In order to apply our theorems, all that is now needed is a moment condition: we suppose that, for some $p>2$ and $w_{p}<\infty$,

$$
\sup _{\alpha_{1} \in \Gamma_{1}} \int_{\Gamma_{2}} \mathrm{E}^{\left(\alpha_{1}, \alpha_{2}\right)}\left|F_{\left(\alpha_{1}, \alpha_{2}\right)}\right|^{p} v_{2}\left(\mathrm{~d} \alpha_{2} \mid \alpha_{1}\right) \leq w_{p}^{p},
$$

where $\mathrm{E}^{\alpha}$ denotes expectation with respect to the Palm distribution, $\mathrm{P}^{\alpha}$, of $H$ at $\alpha$ (Kallenberg (1983, p. 83 and p. 101, Exercise 11.1)).

Theorem 3.2. Under the above conditions, there exists a constant $C \equiv C(d)$ such that, for any $q \leq 3$ and $\rho>0$ satisfying

$$
q<p\left\{1-\frac{1}{\mathrm{e} V(d)(10 \rho)^{d} \kappa}\right\}
$$

we have

$$
\begin{aligned}
& d_{\mathrm{K}}\left(\mathcal{L}\left(\vartheta^{-1}(W-\mathrm{E} W)\right), \mathcal{N}(0,1)\right) \\
& \quad \leq \lambda Q(\rho)+C \lambda\left\{\left(\kappa \rho^{d}\right)^{q-1}\left(w_{p} / \vartheta\right)^{q}+[\lambda Q(\rho)]^{(p-2) / 2 p} w_{p} / \vartheta\right\},
\end{aligned}
$$

where $\vartheta^{2}$ denotes var $W$ and $V(d)$ denotes the volume of the unit ball in d dimensions.

The bound in Theorem 3.2 is explicit, but rather unwieldy. The following two corollaries indicate what can be derived from it, by appropriate choice of $\rho$. They give slight improvements in the exponents on Penrose and Yukich (2005, Theorems 2.3 and 2.5).

Corollary 3.1. Suppose that $Q(\rho) \leq K \mathrm{e}^{-\delta \rho}$ for some $K, \delta>0$. Then, under the conditions of Theorem 3.2, if $\lambda \rightarrow \infty$ with all else fixed,

$$
d_{\mathrm{K}}\left(\mathcal{L}\left(\vartheta^{-1}(W-\mathrm{E} W)\right), \mathcal{N}(0,1)\right)=O\left((\log \lambda)^{d\left(p_{3}-1\right)} \lambda \vartheta^{-p_{3} / 2}\right),
$$

where $p_{3}:=\min \{p, 3\}$.

Corollary 3.2. Suppose that $Q(\rho) \leq K \rho^{-\Delta}$ for some $K>0$, with

$$
\Delta>\frac{2 d\left(p_{3}-1\right)(2 p-1)}{(p-2)\left(p_{3}-2\right)} .
$$

Then, under the conditions of Theorem 3.2, if $\lambda \rightarrow \infty$ with all else fixed and if $\vartheta \asymp \lambda^{1 / 2}$, it follows that

$$
d_{\mathrm{K}}\left(\mathcal{L}\left(\vartheta^{-1}(W-\mathrm{E} W)\right), \mathcal{N}(0,1)\right)=O\left(\lambda^{-\beta}\right),
$$

where

$$
\beta=\frac{p_{3}}{2}-1-\frac{d\left(p_{3}-1\right)\left(p p_{3}-1\right)}{2 d p\left(p_{3}-1\right)+\Delta(p-2)}>0 .
$$


Proof of Theorem 3.2. Fix any $q<p$ such that $q \leq 3$. We aim to apply Corollary 2.2 to $W(\rho)$. A number of the arguments that we use are based on those of Penrose and Yukich (2005).

We begin by bounding $\varepsilon_{4}$, observing first that

$$
R\left(N\left(C_{\alpha}\right)\right)^{q-1}=\left\{\int_{N\left(C_{\alpha}\right)}\left|F_{\gamma}(\rho)\right| H(\mathrm{~d} \gamma)\right\}^{q-1} \leq H\left(N\left(C_{\alpha}\right)\right)^{q-2} \int_{N\left(C_{\alpha}\right)}\left|F_{\gamma}(\rho)\right|^{q-1} H(\mathrm{~d} \gamma)
$$

It follows that

$\operatorname{E~} R\left(N\left(C_{\alpha}\right)\right)^{q-1} \leq \int_{N_{1}\left(C_{\alpha}\right)} \lambda v_{1}\left(\mathrm{~d} \gamma_{1}\right) \int_{\Gamma_{2}} \mathrm{E}^{\left(\gamma_{1}, \gamma_{2}\right)}\left\{\left|F_{\left(\gamma_{1}, \gamma_{2}\right)}(\rho)\right|^{q-1} H\left(N\left(C_{\alpha}\right)\right)^{q-2}\right\} v_{2}\left(\mathrm{~d} \gamma_{2} \mid \gamma_{1}\right)$,

where $N\left(C_{\alpha}\right)=N_{1}\left(C_{\alpha}\right) \times \Gamma_{2}$. Now, for any $\gamma_{1} \in \Gamma_{1}, s, t<p$, and $B \subset \Gamma$, we have

$$
\begin{aligned}
\int_{\Gamma_{2}} \mathrm{E}^{\left(\gamma_{1}, \gamma_{2}\right)}\left\{\left|F_{\gamma}(\rho)\right|^{s} H(B)^{t}\right\} \nu_{2}\left(\mathrm{~d} \gamma_{2} \mid \gamma_{1}\right) \\
\leq\left(\int_{\Gamma_{2}} \mathrm{E}^{\left(\gamma_{1}, \gamma_{2}\right)}\left|F_{\gamma}(\rho)\right|^{p} \nu_{2}\left(\mathrm{~d} \gamma_{2} \mid \gamma_{1}\right)\right)^{s / p} \\
\quad \times\left(\int_{\Gamma_{2}} \mathrm{E}^{\left(\gamma_{1}, \gamma_{2}\right)} H(B)^{p t /(p-s)} \nu_{2}\left(\mathrm{~d} \gamma_{2} \mid \gamma_{1}\right)\right)^{(p-s) / p},
\end{aligned}
$$

by Hölder's inequality. Then, however, $H(B) \sim \mathbf{1}_{B}(\gamma)+\operatorname{Po}(\lambda \nu(B))$ under $\mathrm{P}^{\gamma}$, implying that, from (3.11) and Lemma 4.3,

$$
\int_{\Gamma_{2}} \mathrm{E}^{\left(\gamma_{1}, \gamma_{2}\right)}\left\{\left|F_{\gamma}(\rho)\right|^{s} H(B)^{t}\right\} v_{2}\left(\mathrm{~d} \gamma_{2} \mid \gamma_{1}\right) \leq w_{p}^{s} n^{t}\left\{1+(1.1)^{(p-s) / p}\right\} \leq 2.1 w_{p}^{s} n^{t},
$$

for all $n \in \mathbb{N}$ such that $n \geq \max \{p t /(p-s), 2 \mathrm{e} \lambda v(B)\}$. By applying this inequality to (3.13) with $s=q-1$ and $t=q-2$ and recalling that $N\left(C_{\alpha}\right)=D\left(\alpha_{1}, 10 \lambda^{-1 / d} \rho\right)$, we find that

$$
\operatorname{E~} R\left(N\left(C_{\alpha}\right)\right)^{q-1} \leq 2.1 \lambda v\left(N\left(C_{\alpha}\right)\right) w_{p}^{q-1} n_{\rho}^{q-2} \leq(2.1 / 2 \mathrm{e}) w_{p}^{q-1} n_{\rho}^{q-1},
$$

for

$$
n_{\rho}:=2 \mathrm{e} V(d)(10 \rho)^{d} \kappa,
$$

if we restrict to values of $q \leq 3$ also satisfying (3.12), since, with the above choices of $s$ and $t$ and for such $q$,

$$
\frac{p t}{p-s}<\frac{p(q-1)}{p-q} \leq n_{\rho}
$$

and $\lambda \nu\left(N\left(C_{\alpha}\right)\right) \leq n_{\rho} / 2 \mathrm{e}$. It then follows immediately that

$$
\begin{aligned}
\varepsilon_{4} & =\vartheta(\rho)^{-q} \int_{\Gamma} \operatorname{E} R\left(N\left(C_{\alpha}\right)\right)^{q-1} \operatorname{E} R(\mathrm{~d} \alpha) \\
& \leq \vartheta(\rho)^{-q} \int_{\Gamma}\{2.1 / 2 \mathrm{e}\} w_{p}^{q-1} n_{\rho}^{q-1} w_{p} \lambda \nu(\mathrm{d} \alpha) \\
& \leq \frac{1}{2} \lambda \vartheta(\rho)^{-q} w_{p}^{q} n_{\rho}^{q-1},
\end{aligned}
$$


where $\vartheta(\rho)$ is the standard deviation of $W(\rho)$. For $\varepsilon_{3}$, we observe that

$$
\begin{aligned}
\mathrm{E} \int_{\Gamma} & R\left(N\left(C_{\alpha}\right)\right)^{q-1} R(\mathrm{~d} \alpha) \\
\leq & \mathrm{E}\left\{\int_{\alpha \in \Gamma}\left|F_{\alpha}(\rho)\right| H\left(N\left(C_{\alpha}\right)\right)^{q-2} \int_{\gamma \in N\left(C_{\alpha}\right)}\left|F_{\gamma}(\rho)\right|^{q-1} H(\mathrm{~d} \gamma) H(\mathrm{~d} \alpha)\right\} \\
\leq & \mathrm{E} \int_{\Gamma}\left|F_{\alpha}(\rho)\right|^{q} H\left(N\left(C_{\alpha}\right)\right)^{q-2} H(\mathrm{~d} \alpha) \\
& +\mathrm{E} \int_{\alpha \in \Gamma} \int_{\substack{\gamma \in N\left(C_{\alpha}\right) \\
\gamma \neq \alpha}}\left(\left|F_{\alpha}(\rho)\right|^{q}+\left|F_{\gamma}(\rho)\right|^{q}\right) H\left(N\left(C_{\alpha}\right)\right)^{q-2} H(\mathrm{~d} \gamma) H(\mathrm{~d} \alpha) .
\end{aligned}
$$

The first expectation in (3.16) is bounded by taking $s=q$ and $t=q-2$ in (3.14), giving at most $2.1 \lambda w_{p}^{q} n_{\rho}^{q-2}$; the first half of the second expectation follows by taking $s=q$ and $t=q-1$ in (3.14), giving at most $2.1 \lambda w_{p}^{q} n_{\rho}^{q-1}$; and the remaining term is at most

$$
\mathrm{E} \int_{\Gamma}\left|F_{\gamma}(\rho)\right|^{q} H\left(D\left(\gamma_{1}, 20 \lambda^{-1 / d} \rho\right)\right)^{q-1} H(\mathrm{~d} \gamma),
$$

bounded in the same way by $2.1 \lambda w_{p}^{q}\left(2^{d} n_{\rho}\right)^{q-1}$. It follows that

$$
\varepsilon_{3} \leq 2.1 \lambda \vartheta(\rho)^{-q} w_{p}^{q} n_{\rho}^{q-1}\left(2^{d(q-1)}+2\right) .
$$

For the remaining element, $\varepsilon_{5}$, of the error in Corollary 2.2, we note that, for any $\alpha \in \Gamma$,

$$
\mathrm{E} R\left(N\left(C_{\alpha}\right)\right) \leq w_{p} \lambda v\left(N\left(C_{\alpha}\right)\right) \leq w_{p} n_{\rho} / 2 \mathrm{e},
$$

giving

$$
\varepsilon_{5} \leq \vartheta(\rho)^{-1} \mathrm{e}^{-1} w_{p} n_{\rho}
$$

In order to show that this is comparable with the errors $\varepsilon_{3}$ and $\varepsilon_{4}$, we now need to bound $\vartheta(\rho)$. To do so, observe that

$$
\begin{aligned}
\vartheta(\rho)^{2} & =\mathrm{E} \int_{\alpha \in \Gamma} \int_{\gamma \in A_{\alpha}}\left(F_{\alpha}(\rho) H(\mathrm{~d} \alpha)-\bar{F}_{\alpha}(\rho) \mu(\mathrm{d} \alpha)\right)\left(F_{\gamma}(\rho) H(\mathrm{~d} \gamma)-\bar{F}_{\gamma}(\rho) \mu(\mathrm{d} \gamma)\right) \\
& \leq \mathrm{E} \int_{\alpha \in \Gamma} \int_{\gamma \in A_{\alpha}}(R(\mathrm{~d} \alpha)+\mathrm{E} R(\mathrm{~d} \alpha))(R(\mathrm{~d} \gamma)+\mathrm{E} R(\mathrm{~d} \gamma)) \\
& \leq \mathrm{E} \int_{\alpha \in \Gamma} \int_{\gamma \in A_{\alpha}} R\left(A_{\alpha}\right) R(\mathrm{~d} \alpha)+3 \int_{\Gamma} \mathrm{E} R\left(A_{\alpha}\right) \mathrm{E} R(\mathrm{~d} \alpha) .
\end{aligned}
$$

The second of these quantities is immediately bounded by

$$
3 \lambda \int_{\Gamma} w_{p} v(\mathrm{~d} \alpha) \frac{w_{p} n_{\rho}}{5^{d} \times 2 \mathrm{e}} \leq \frac{\lambda n_{\rho} w_{p}^{2}}{9} .
$$

For the first, arguing as in (3.16), but with $q$ replaced by 2 and with $A_{\alpha}$ in place of $N\left(C_{\alpha}\right)$, we obtain the bound

$$
\lambda w_{p}^{2}+2.1 \lambda w_{p}^{2} n_{\rho}\left\{\left(\frac{1}{5}\right)^{d}+\left(\frac{2}{5}\right)^{d}\right\} .
$$

By adding the two quantities, and recalling that $n_{\rho} \geq 1$, we find that

$$
\vartheta(\rho)^{2} \leq 2.5 \lambda w_{p}^{2} n_{\rho}
$$


Thus, it follows from (3.18) that

$$
\begin{aligned}
\varepsilon_{5} & \leq \vartheta(\rho)^{-q} \mathrm{e}^{-1} w_{p} n_{\rho}\left\{2.5 \lambda w_{p}^{2} n_{\rho}\right\}^{(q-1) / 2} \\
& \leq \vartheta(\rho)^{-q} w_{p}^{q} \lambda n_{\rho}^{q-1}\left\{n_{\rho} / \lambda\right\}^{(3-q) / 2} \\
& \leq \lambda \vartheta(\rho)^{-q} w_{p}^{q} n_{\rho}^{q-1}
\end{aligned}
$$

provided that $n_{\rho} \leq \lambda$; if this is not the case, then it already follows from (3.19) that $\lambda \vartheta(\rho)^{-q} n_{\rho}^{q-1}$ is large, implying that the bound is in any case meaningless. Hence, $\varepsilon_{5}$ is indeed bounded in (3.20) by a quantity of the same order as those in (3.15) and (3.17).

However, the argument is not yet finished, since applying Corollary 2.2 to $W(\rho)$ leaves $\vartheta(\rho)$ rather than $\vartheta$ in the denominator, and the difference is a major contributor to the error bound. Writing $E^{*}$ for the event $\{W \neq W(\rho)\}$, of probability at most $\lambda Q(\rho)$, we use Hölder's inequality to show that

$$
\begin{aligned}
\mathrm{E}(W-W(\rho))^{2} & =\mathrm{E}\left\{(W-W(\rho))^{2} \mathbf{1}_{E^{*}}\right\} \\
& \leq\left(\mathrm{E}|W-W(\rho)|^{p}\right)^{2 / p}\left(\mathrm{P}\left(E^{*}\right)\right)^{(p-2) / p} \\
& \leq 2\left\{\left(\mathrm{E}|W|^{p}\right)^{2 / p}+\left(\mathrm{E}|W(\rho)|^{p}\right)^{2 / p}\right\}\left(\mathrm{P}\left(E^{*}\right)\right)^{(p-2) / p} .
\end{aligned}
$$

Now, both $\mathrm{E}|W|^{p}$ and $\mathrm{E}|W(\rho)|^{p}$ are bounded by

$$
\begin{aligned}
\mathrm{E}\left(\int_{\Gamma}\left|F_{\alpha}\right| H(\mathrm{~d} \alpha)\right)^{p} & \leq \mathrm{E}\left\{H(\Gamma)^{p-1} \int_{\Gamma}\left|F_{\alpha}\right|^{p} H(\mathrm{~d} \alpha)\right\} \\
& \leq 2.1 w_{p}^{p}(2 \mathrm{e} \lambda)^{p-1} \lambda \\
& \leq 8.4 \mathrm{e}^{2}\left(\lambda w_{p}\right)^{p}
\end{aligned}
$$

as can be seen by applying (3.14) with $s=p, t=p-1$, and $B=\Gamma$. Thus,

$$
\mathrm{E}(W-W(\rho))^{2} \leq 4\left(8.4 \mathrm{e}^{2}\right)^{2 / p}\left(\lambda w_{p}\right)^{2}[\lambda Q(\rho)]^{(p-2) / p} .
$$

This in turn implies that

$$
\begin{aligned}
\vartheta^{-2}\left|\vartheta^{2}-\vartheta(\rho)^{2}\right| & \leq \vartheta^{-2}\{2|\operatorname{cov}(W-W(\rho), W)|+\operatorname{var}(W-W(\rho))\} \\
& \leq 2 x_{\lambda, \rho}\left(1+x_{\lambda, \rho}\right),
\end{aligned}
$$

where $x_{\lambda, \rho}:=2\left(8.4 \mathrm{e}^{2}\right)^{1 / p} \lambda w_{p}[\lambda Q(\rho)]^{(p-2) / 2 p}$. Recall that $d_{\mathrm{K}}(\mathcal{N}(0,1), \mathcal{N}(0,1+\varepsilon)) \leq$ $\varepsilon /(2 \sqrt{2 \pi})$. It follows that, in changing the denominator from var $W(\rho)$ to var $W$, a further error of at most $(1 / \sqrt{2 \pi}) x_{\lambda, \rho}\left(1+x_{\lambda, \rho}\right) \leq x_{\lambda, \rho}$ is incurred (again since the bound is trivial if $\left.x_{\lambda, \rho} \geq 1\right)$. This completes the proof of the theorem.

The corollaries are proved by substituting appropriate values for $\rho$ into the explicit bound given by the theorem. For Corollary 3.1, take $\rho=k \delta^{-1} \log \lambda$ for $k>7$ and take $q$ to be the largest value consistent with (3.12). Then note that if $p \leq 3$, this value, $q \equiv q(\lambda)$, approaches $p$ fast enough as $\lambda \rightarrow \infty$ for $(\operatorname{var} W)^{q}$ to be asymptotically equivalent to $(\operatorname{var} W)^{p}$. For Corollary 3.2, take $\rho=\lambda^{\beta^{\prime}}$, where

$$
\beta^{\prime}:=\frac{p q-1}{2 d p(q-1)+\Delta(p-2)}
$$

and $q$ is again the largest value consistent with (3.12). 


\subsection{Maximal points}

Let $W$ be the number of maximal points of a Poisson process $H$ of rate $\lambda$ in a region

$$
D:=\{(x, y): 0 \leq x \leq 1,0 \leq y \leq f(x)\}
$$

where $f$ is absolutely continuous, decreasing, and such that $f(0)=1, f(1)=0$, and

$$
m_{1}:=\underset{0 \leq x \leq 1}{\operatorname{ess} \inf }\left|f^{\prime}(x)\right|>0, \quad m_{2}:=\underset{0 \leq x \leq 1}{\operatorname{ess} \sup }\left|f^{\prime}(x)\right|<\infty .
$$

A point $\alpha=(x, y)$ of $H$ is maximal if $H\left(D_{\alpha}\right)=0$, where

$$
D_{\alpha}:=\left\{(u, v): x \leq u \leq f^{-1}(y), y \leq v \leq f(u)\right\} \backslash\{\alpha\} .
$$

Hence,

$$
W=\int_{D} H(\mathrm{~d} \alpha) \mathbf{1}_{\left\{H\left(D_{\alpha}\right)=0\right\}}=: \int_{D} \Xi(\mathrm{d} \alpha)
$$

is a random variable of the form considered in this paper, with $F_{\alpha}=\mathbf{1}_{\left\{H\left(D_{\alpha}\right)=0\right\}} \geq 0$ and, hence, $R=\Xi$. However, the asymptotic structure is rather different from that in the previous section, necessitating separate arguments.

There have been a number of papers contributing to the central limit theorem for $W$, under a variety of conditions on the function $f$. With $\mu_{2}$ the mean measure of $\Xi$,

$$
\mu_{2}(\mathrm{~d} \alpha):=\mathrm{E}\left\{H(\mathrm{~d} \alpha) \mathbf{1}_{\left\{H\left(D_{\alpha}\right)=0\right\}}\right\}=\lambda \mathrm{e}^{-\lambda\left|D_{\alpha}\right|} \mathrm{d} \alpha,
$$

the asymptotics of the first and second moments, as $\lambda \rightarrow \infty$, are given by (Devroye (1993), Bai et al. (1998))

$$
\begin{aligned}
\mu_{2}(D)=\mathrm{E} \Xi(D) & \sim \lambda^{1 / 2} \sqrt{\frac{\pi}{2}} \int_{0}^{1}\left|f^{\prime}(x)\right|^{1 / 2} \mathrm{~d} x, \\
& \operatorname{var} \Xi(D) \sim(2 \log 2-1) \mu_{2}(D) .
\end{aligned}
$$

Central limit theorems are given in Bai et al. (2001) and in Barbour and Xia (2001); in the latter paper, Stein's method is used to give a rate of convergence with respect to the bounded Wasserstein distance. Here, we prove error bounds with respect to the Kolmogorov distance, using some of the same ideas, but now applying Corollary 2.2 to provide the bound in the stronger metric. The case in which $D$ is the unit square, which does not fit our assumptions, has quite different, logarithmic asymptotics for the moments, and is actually a classical record value problem. The unit cube in higher dimensions has been considered separately in Baryshnikov (2000) and in Bai et al. (2005); the latter paper again uses Stein's method.

Theorem 3.3 gives a rate of convergence under the above conditions on $f$. In Theorem 3.4, we relax the conditions on $f$ to allow for natural regions, such as the quarter circle, whose boundaries may be flat or vertical at 0 or 1 .

Theorem 3.3. Under the above conditions on $f$,

$$
d_{\mathrm{K}}\left(\mathcal{L}\left(\frac{W-\mathrm{E} W}{\sqrt{\operatorname{var} W}}\right), \mathcal{N}(0,1)\right)=O\left(\lambda^{-1 / 4} \log \lambda\right) .
$$


Proof. In order to find neighbourhoods of local dependence, we begin by truncating the set $D$ (see Barbour and Xia (2001, Lemma 3.1) and Bai et al. (2005)), replacing $W$ by $\tilde{W}:=$ $\int_{D_{\lambda}^{*}} \Xi(\mathrm{d} \alpha)$, where

$$
D_{\lambda}^{*}=\left\{(x, y): 0 \leq x \leq 1, f_{\lambda}(x) \leq y \leq f(x)\right\} \subset D
$$

and

$$
f_{\lambda}(x):=\inf \left\{y \geq 0:\left|D_{(x, y)}\right| \leq 4 \lambda^{-1} \log \lambda\right\} .
$$

Since $\mu_{2}(\mathrm{~d} \alpha) \leq \lambda^{-3} \mathrm{~d} \alpha$ if $\alpha \in D \backslash D_{\lambda}^{*}$, it follows that $\mathrm{P}(W \neq \tilde{W}) \leq \lambda^{-3}$ and that, as for (3.21),

$$
\begin{aligned}
\mathrm{E}(W-\tilde{W})^{2} & \leq \mathrm{E}\left\{H^{2}\left(D \backslash D_{\lambda}^{*}\right) \mathbf{1}_{\{W \neq \tilde{W}\}}\right\} \\
& \leq\left\{2.1(2 \mathrm{e} \lambda)^{6}\right\}^{1 / 3}\left(\lambda^{-3}\right)^{2 / 3} \\
& =O(1),
\end{aligned}
$$

from Lemma 4.3; hence, as in (3.22),

$$
\vartheta^{-2}|\operatorname{var} W-\operatorname{var} \tilde{W}| \leq 2 x(1+x)
$$

with $x=\vartheta^{-1} \sqrt{\mathrm{E}\left\{(W-\tilde{W})^{2}\right\}}=O\left(\lambda^{-1 / 4}\right)$, enabling $W$ to be replaced by $\tilde{W}$ to the accuracy that we require.

We then write

$$
g(x):=f_{\lambda}^{-1}(f(x)), \quad h(y):=f_{\lambda}\left(f^{-1}(y)\right),
$$

where $f_{\lambda}^{-1}(y):=0$ if $y>f_{\lambda}(0)$, and take

$$
\begin{aligned}
A_{\alpha} & :=\left\{(u, v): u \leq f^{-1}(y), v \leq f(x)\right\} \cap D_{\lambda}^{*}, \\
B_{\alpha} & :=\left\{(u, v): u \leq f^{-1}(h(y)), v \leq f(g(x))\right\} \cap D_{\lambda}^{*}, \\
C_{\alpha} & :=\left\{(u, v): u \leq f^{-1}\left(h^{(2)}(y)\right), v \leq f\left(g^{(2)}(x)\right)\right\} \cap D_{\lambda}^{*}, \\
N\left(C_{\alpha}\right) & :=\left\{(u, v): u \leq f^{-1}\left(h^{(4)}(y)\right), v \leq f\left(g^{(4)}(x)\right)\right\} \cap D_{\lambda}^{*},
\end{aligned}
$$

where $\varphi^{(j)}$ denotes the $j$ th iterate of the function $\varphi$. These neighbourhoods meet the requirements of condition (LD3) because of the independence properties of the Poisson process $H$. Applying Corollary 2.2, since $\vartheta^{2}=\operatorname{var} W \asymp \lambda^{1 / 2}$, from (3.24), we see that the error in the normal approximation to $\tilde{W}$ is of order $O\left(\varepsilon_{3}^{\prime}+\varepsilon_{4}^{\prime}+\varepsilon_{5}^{\prime}\right)$, where

$$
\begin{aligned}
& \varepsilon_{3}^{\prime}:=\lambda^{-3 / 4} \int_{D_{\lambda}^{*}} \mu_{2}(\mathrm{~d} \alpha) \mathrm{E}^{\alpha} \Xi^{2}\left(N\left(C_{\alpha}\right)\right), \\
& \varepsilon_{4}^{\prime}:=\lambda^{-3 / 4} \int_{D_{\lambda}^{*}} \mu_{2}(\mathrm{~d} \alpha) \mathrm{E} \Xi^{2}\left(N\left(C_{\alpha}\right)\right), \\
& \varepsilon_{5}^{\prime}:=\lambda^{-1 / 4} \sup _{\alpha \in D_{\lambda}^{*}} \mu_{2}\left(N\left(C_{\alpha}\right)\right) .
\end{aligned}
$$

Consider $\varepsilon_{4}^{\prime}$. We note that

$$
N\left(C_{\alpha}\right) \subset D_{\left(g^{(5)}(x), h^{(5)}(y)\right)}
$$


and that $D_{(u, v)}$, suitably scaled, is a region of the same form as the original region $D$. Indeed, the number of maximal points in $D_{(u, v)}$ has the same distribution as the number of maximal points in the region

$$
D^{\prime}:=\left\{(r, s): 0 \leq r \leq 1,0 \leq s \leq \varphi_{u, v}(r)\right\},
$$

where

$$
\varphi_{u, v}(r):=\frac{f\left(r f^{-1}(v)+(1-r) u\right)-v}{f(u)-v},
$$

for an underlying Poisson process with intensity

$$
\lambda^{\prime}:=\lambda\left(f^{-1}(v)-u\right)(f(u)-v) .
$$

Thus, (3.23) and (3.24) give the asymptotic formulae

$$
\begin{aligned}
\mathrm{E} \Xi\left(D_{(u, v)}\right) & \sim \sqrt{\lambda^{\prime}} \sqrt{\frac{\pi}{2}} \int_{0}^{1}\left|\varphi_{u, v}^{\prime}(r)\right|^{1 / 2} \mathrm{~d} r, \\
\mathrm{E} \Xi^{2}\left(D_{(u, v)}\right) & \sim\left(\mathrm{E} \Xi\left(D_{(u, v)}\right)\right)^{2}+(2 \log 2-1) \mathrm{E} \Xi\left(D_{(u, v)}\right),
\end{aligned}
$$

and, so, we need only consider the asymptotics of (3.27).

To do so, note that

$$
\int_{0}^{1}\left|\varphi_{u, v}^{\prime}(r)\right|^{1 / 2} \mathrm{~d} r=\frac{\int_{u}^{f^{-1}(v)}\left|f^{\prime}(w)\right|^{1 / 2} \mathrm{~d} w}{\sqrt{\left(f^{-1}(v)-u\right)(f(u)-v)}}
$$

so that

$$
\mathrm{E} \Xi\left(D_{(u, v)}\right) \sim \sqrt{\frac{\pi \lambda}{2}} \int_{u}^{f^{-1}(v)}\left|f^{\prime}(w)\right|^{1 / 2} \mathrm{~d} w=: m(u, v),
$$

say. In order to estimate $m(u, v)$ with $(u, v)=\left(g^{(5)}(x), h^{(5)}(y)\right)$, we now observe, from the definition of $f_{\lambda}$, that $\frac{1}{2} m_{1}(x-g(x))^{2} \leq 4 \lambda^{-1} \log \lambda$ for any $x$, implying that

$$
0 \leq x-g(x) \leq 2 \sqrt{\frac{2 \log \lambda}{\lambda m_{1}}}
$$

and, hence, by iteration, that

$$
0 \leq x-g^{(5)}(x) \leq 10 \sqrt{\frac{2 \log \lambda}{\lambda m_{1}}} .
$$

It similarly follows that

$$
f^{-1}\left(h^{(5)}(y)\right)-f^{-1}(y) \leq 10 \sqrt{\frac{2 \log \lambda}{\lambda m_{1}}}, \quad 0 \leq y-h^{(5)}(y) \leq 10 \sqrt{\frac{2 m_{2} \log \lambda}{\lambda}},
$$

and that, for $(x, y) \in D_{\lambda}^{*}$,

$$
f^{-1}(y)-x \leq 2 \sqrt{\frac{2 \log \lambda}{\lambda m_{1}}}, \quad 0 \leq f(x)-y \leq 2 \sqrt{\frac{2 m_{2} \log \lambda}{\lambda}} .
$$


Hence, for $(x, y) \in D_{\lambda}^{*}$,

$$
\begin{aligned}
\sqrt{\frac{2}{\pi}} m\left(g^{(5)}(x), h^{(5)}(y)\right) & \leq \sqrt{\lambda m_{2}}\left(f^{-1}\left(h^{(5)}(y)\right)-g^{(5)}(x)\right) \\
& \leq 22 \sqrt{2 \log \lambda} \sqrt{m_{2} / m_{1}} .
\end{aligned}
$$

It thus follows easily from (3.23), (3.27), (3.28), (3.29), and (3.33) that

$$
\lambda^{3 / 4} \varepsilon_{4}^{\prime}=\int_{D_{\lambda}^{*}} \mu_{2}(\mathrm{~d} \alpha) \mathrm{E} \Xi^{2}\left(N\left(C_{\alpha}\right)\right)=O\left(\lambda^{1 / 2} \log \lambda\right) .
$$

To calculate $\varepsilon_{3}^{\prime}$, we need to bound $\mathrm{E}^{\alpha} \Xi^{2}\left(N\left(C_{\alpha}\right)\right)$. We begin by observing that, under the measure $\mathrm{P}^{\alpha}$,

$$
\Xi\left(D_{\alpha}\right)=\Xi([0, x] \times[0, y] \backslash\{\alpha\})=0 \quad \text { almost surely }
$$

when $\alpha=(x, y)$. Hence,

$$
\Xi\left(N\left(C_{\alpha}\right)\right) \leq \Xi\left(N_{U}\left(C_{\alpha}\right)\right)+\Xi\left(N_{L}\left(C_{\alpha}\right)\right),
$$

a sum of two independent components, where

$$
\begin{aligned}
& N_{U}\left(C_{\alpha}\right):=D_{\left(g^{(5)}(x), y\right)} \cap\{[0, x) \times(y, 1]\}, \\
& N_{L}\left(C_{\alpha}\right):=D_{\left(x, h^{(5)}(y)\right)} \cap\{(x, 1] \times[0, y)\} .
\end{aligned}
$$

However, we cannot immediately deduce the asymptotics of the moments of $\Xi\left(N_{U}\left(C_{\alpha}\right)\right)$ and $\Xi\left(N_{L}\left(C_{\alpha}\right)\right)$ by scaling using (3.23) and (3.24), because the former region has a vertical section in its upper-right boundary and the latter a horizontal section.

To circumvent this problem, we split each region into two pieces. For $N_{U}\left(C_{\alpha}\right)$, we define

$$
D_{2 U}:=N_{U}\left(C_{\alpha}\right) \cap\left\{(u, v): 2 m_{2} u+v \geq 2 m_{2} x+y\right\} \cap\left\{(u, v): m_{2} u+v \leq m_{2} x+f(x)\right\},
$$

and set $D_{1 U}:=N_{U}\left(C_{\alpha}\right) \backslash D_{2 U}$. Then $D_{1 U}$ is also a scaled version of a region of the same form as $D$, but now with boundary function $\varphi$ having $m_{1} \leq\left|\varphi^{\prime}\right| \leq 2 m_{2}$, and

$$
\Xi\left(N_{U}\left(C_{\alpha}\right)\right) \leq \Xi^{U}\left(D_{1 U}\right)+H\left(D_{2 U}\right),
$$

where $\Xi^{U}$ is the process of points maximal in $D_{1 U}$ :

$$
\Xi^{U}(\mathrm{~d} \beta)=H(\mathrm{~d} \beta) \mathbf{1}_{\left\{H\left(D_{\beta} \backslash D_{2 U}\right)=0\right\}} .
$$

Note that $\Xi^{U}\left(D_{1 U}\right)$ and $H\left(D_{2 U}\right)$ are independent. Arguing analogously for $\Xi\left(N_{L}\left(C_{\alpha}\right)\right)$, we obtain

$$
\begin{aligned}
\mathrm{E}^{\alpha} \Xi^{2}\left(N\left(C_{\alpha}\right)\right) \leq & \mathrm{E}\left(\Xi^{U}\left(D_{1 U}\right)+H\left(D_{2 U}\right)+\Xi^{L}\left(D_{1 L}\right)+H\left(D_{2 L}\right)\right)^{2} \\
\leq & {\left[\mathrm{E}\left(\Xi^{U}\left(D_{1 U}\right)+H\left(D_{2 U}\right)+\Xi^{L}\left(D_{1 L}\right)+H\left(D_{2 L}\right)\right)\right]^{2} } \\
& +\operatorname{var} \Xi^{U}\left(D_{1 U}\right)+\operatorname{var} H\left(D_{2 U}\right)+\operatorname{var} \Xi^{L}\left(D_{1 L}\right)+\operatorname{var} H\left(D_{2 L}\right) .
\end{aligned}
$$

We now observe that

$$
\left|D_{2 U}\right| \leq \frac{1}{2 m_{2}}(f(x)-y)^{2}, \quad\left|D_{2 L}\right| \leq \frac{m_{1}}{2}\left(f^{-1}(y)-x\right)^{2} \leq \frac{1}{2 m_{1}}(f(x)-y)^{2} .
$$


Hence, we have

$$
\begin{aligned}
& \mathrm{E} H\left(D_{2 U}\right)=\operatorname{var} H\left(D_{2 U}\right) \leq \frac{\lambda}{2 m_{2}}(f(x)-y)^{2}, \\
& \mathrm{E} H\left(D_{2 L}\right)=\operatorname{var} H\left(D_{2 L}\right) \leq \frac{\lambda}{2 m_{1}}(f(x)-y)^{2},
\end{aligned}
$$

whereas, as for (3.33) above,

$$
\mathrm{E} \Xi^{U}\left(D_{1 U}\right)+\operatorname{var} \Xi^{U}\left(D_{1 U}\right)+\mathrm{E} \Xi^{L}\left(D_{1 L}\right)+\operatorname{var} \Xi^{L}\left(D_{1 L}\right)=O\left(\sqrt{\left(m_{2} / m_{1}\right) \log \lambda}\right) .
$$

Now, however,

$$
\mu_{2}(\mathrm{~d} \alpha) \leq \lambda \exp \left\{-\frac{\lambda}{2 m_{2}}(f(x)-y)^{2}\right\} \mathrm{d} \alpha,
$$

and, hence, by integration,

$$
\begin{aligned}
\lambda^{3 / 4} \varepsilon_{3}^{\prime} & =\int_{D_{\lambda}^{*}} \mu_{2}(\mathrm{~d} \alpha) \mathrm{E}^{\alpha} \Xi^{2}\left(N\left(C_{\alpha}\right)\right) \\
& =O\left(\lambda^{1 / 2}\left(m_{2} / m_{1}\right)\left\{\log \lambda+\left(m_{2} / m_{1}\right) \sqrt{m_{2}}\right\}\right) \\
& =O\left(\lambda^{1 / 2} \log \lambda\right) .
\end{aligned}
$$

Finally, it follows from (3.26), (3.29), and (3.33) that

$$
\lambda^{1 / 4} \varepsilon_{5}^{\prime}=\sup _{\alpha \in D_{\lambda}^{*}} \mu_{2}\left(N\left(C_{\alpha}\right)\right)=O(\sqrt{\log \lambda}),
$$

and this, combined with (3.34), (3.39), and (3.25), proves the theorem.

If $m_{1}=0$ or $m_{2}=\infty$, then the argument needs modification. However, the changes needed may frequently not be too elaborate, since the contribution to the integrals in (3.25) from any region $D_{\lambda}^{*} \cap\{[a, b] \times[0,1]\}$, where

$$
0<\underset{a \leq x \leq b}{\operatorname{ess} \inf }\left|f^{\prime}(x)\right| \leq \underset{a \leq x \leq b}{\operatorname{ess} \sup }\left|f^{\prime}(x)\right|<\infty,
$$

is already of order $O\left(\lambda^{-1 / 4} \log \lambda\right)$, by the previous argument. To illustrate the alterations needed, we now suppose that (3.40) is true for some $a$ and $b, 0<a<b<1$, and that

$$
\begin{aligned}
& 0<\tau_{1}:=\underset{0<x \leq(2 a \wedge 1)}{\operatorname{ess} \inf } x^{-\beta}\left|f^{\prime}(x)\right| \leq \underset{0<x \leq(2 a \wedge 1)}{\operatorname{ess} \sup } x^{-\beta}\left|f^{\prime}(x)\right|=: \tau_{2}<\infty,
\end{aligned}
$$

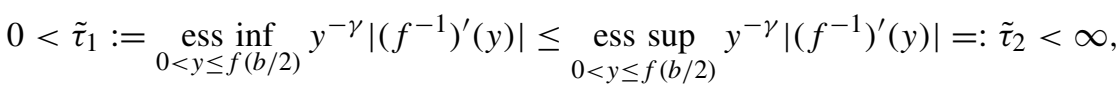

for some $\beta, \gamma>-1$.

Theorem 3.4. If $f$ is decreasing, with $f(0)=1$ and $f(1)=0$, if (3.40) is true for some a and $b, 0<a<b<1$, and if (3.41) and (3.42) also hold, then

$$
d_{\mathrm{K}}\left(\mathcal{L}\left(\frac{W-\mathrm{E} W}{\sqrt{\operatorname{var} W}}\right), \mathcal{N}(0,1)\right)=O\left(\lambda^{-1 / 4} \log \lambda\right) .
$$


Proof. The estimates (3.30)-(3.32) and (3.35)-(3.38) are essentially local in character. For any fixed $C_{0}>1$, they hold for any $(x, y) \in D_{\lambda}^{*}$, with $m_{1}$ replaced by $\left(1 / C_{0}\right)\left|f^{\prime}(x)\right|$ and $m_{2}$ replaced by $C_{0}\left|f^{\prime}(x)\right|$; thus, (3.33) and (3.37) also hold, with $m_{2} / m_{1}$ replaced by $C_{0}^{2}$, provided that

$$
\frac{1}{C_{0}}\left|f^{\prime}(x)\right| \leq\left|f^{\prime}(z)\right| \leq C_{0}\left|f^{\prime}(x)\right| \text { for all } g^{(5)}(x) \leq z \leq f^{-1}\left(h^{(5)}(y)\right) .
$$

In turn, this holds provided that

$$
\frac{1}{C_{0}}\left|f^{\prime}(x)\right| \leq\left|f^{\prime}(z)\right| \leq C_{0}\left|f^{\prime}(x)\right| \text { for all }|z-x| \leq 12 \sqrt{\frac{2 C_{0} \log \lambda}{\lambda\left|f^{\prime}(x)\right|}} .
$$

We concentrate now on pairs $\alpha=(x, y) \in D_{\lambda}^{*}$ in which $x$ is small, since the argument for values of $x$ near 1 is entirely symmetrical. First, for $0 \leq x, z \leq(1 \wedge 3 a / 2)$, from (3.41) we have

$$
\frac{\tau_{1}}{\tau_{2}}\left(1-\frac{|z-x|}{x}\right)^{\beta} \leq \frac{\left|f^{\prime}(z)\right|}{\left|f^{\prime}(x)\right|} \leq \frac{\tau_{1}}{\tau_{2}}\left(1+\frac{|z-x|}{x}\right)^{\beta},
$$

meaning that, with $C_{0}=2^{\beta} \tau_{2} / \tau_{1},(3.43)$ can only be violated for $x$ such that

$$
\frac{12}{x} \sqrt{\frac{2 C_{0} \log \lambda}{\lambda\left|f^{\prime}(x)\right|}}>\frac{1}{2} \text {. }
$$

However, this requires that

$$
x^{2}\left|f^{\prime}(x)\right|<1152 C_{0} \lambda^{-1} \log \lambda,
$$

and, from (3.41) and for $\lambda$ large enough, this can only be the case if

$$
x<x_{\lambda}:=k\left\{\lambda^{-1} \log \lambda\right\}^{1 /(2+\beta)},
$$

for an appropriately chosen $k$. This, together with the corresponding argument for values of $x$ near 1 , shows both that the contributions to $\varepsilon_{3}^{\prime}$ and $\varepsilon_{4}^{\prime}$ from

$$
J_{\lambda}:=D_{\lambda}^{*} \cap\left\{\left[x_{\lambda}, 1-x_{\lambda}^{\prime}\right] \times[0,1]\right\}
$$

are still of order $O\left(\lambda^{1 / 4} \log \lambda\right)$, where $1-x_{\lambda}^{\prime}=f^{-1}\left(k^{\prime}\left(\lambda^{-1} \log \lambda\right)^{1 /(2+\gamma)}\right)$ for some suitably chosen $k^{\prime}$, and that, with reference to $\varepsilon_{5}^{\prime}$,

$$
\sup _{\alpha \in J_{\lambda}} \mu_{2}\left(N\left(C_{\alpha}\right)\right)=O(\sqrt{\log \lambda}) .
$$

It remains to consider pairs $\alpha=(x, y) \in D_{\lambda}^{*}$ such that $x \leq x_{\lambda}$ or $x \geq 1-x_{\lambda}^{\prime}$; again, we only give the argument for small $x$. For $\alpha=(x, y) \in D_{\lambda}^{*}$ such that $x \leq x_{\lambda}$, it is necessarily the case that $y \geq y_{\lambda}:=f_{\lambda}\left(x_{\lambda}\right)$ and, hence, that $h^{(5)}(y) \geq h^{(5)}\left(y_{\lambda}\right)$ and $f^{-1}\left(h^{(5)}(y)\right) \leq f^{-1}\left(h^{(5)}\left(y_{\lambda}\right)\right)$. By applying (3.31) and (3.32) at $\left(x_{\lambda}, y_{\lambda}\right)$ with $m_{1}$ replaced by $\left(1 / C_{0}\right)\left|f^{\prime}\left(x_{\lambda}\right)\right|$ and $m_{2}$ replaced by $C_{0}\left|f^{\prime}\left(x_{\lambda}\right)\right|$, we thus obtain

$$
\begin{aligned}
f\left(x_{\lambda}\right)-y_{\lambda} & \leq 2 \sqrt{\frac{2 C_{0} \log \lambda}{\lambda}\left|f^{\prime}\left(x_{\lambda}\right)\right|}, & y_{\lambda}-h^{(5)}\left(y_{\lambda}\right) & \leq 10 \sqrt{\frac{2 C_{0} \log \lambda}{\lambda}\left|f^{\prime}\left(x_{\lambda}\right)\right|}, \\
f^{-1}\left(y_{\lambda}\right)-x_{\lambda} & \leq 2 \sqrt{\frac{2 C_{0} \log \lambda}{\lambda\left|f^{\prime}\left(x_{\lambda}\right)\right|}}, & f^{-1}\left(h^{(5)}\left(y_{\lambda}\right)\right)-f^{-1}\left(y_{\lambda}\right) & \leq 10 \sqrt{\frac{2 C_{0} \log \lambda}{\lambda\left|f^{\prime}\left(x_{\lambda}\right)\right|}} .
\end{aligned}
$$


Thus,

$$
\begin{aligned}
1-h^{(5)}\left(y_{\lambda}\right) & \leq 1-f\left(x_{\lambda}\right)+12 \sqrt{\frac{2 C_{0} \log \lambda}{\lambda}\left|f^{\prime}\left(x_{\lambda}\right)\right|,} \\
f^{-1}\left(h^{(5)}\left(y_{\lambda}\right)\right) & \leq x_{\lambda}+12 \sqrt{\frac{2 C_{0} \log \lambda}{\lambda\left|f^{\prime}\left(x_{\lambda}\right)\right|}},
\end{aligned}
$$

and also, from (3.41),

$$
1-f\left(x_{\lambda}\right) \leq \tau_{2} x_{\lambda}^{\beta+1}, \quad \tau_{1} x_{\lambda}^{\beta} \leq\left|f^{\prime}\left(x_{\lambda}\right)\right| \leq \tau_{2} x_{\lambda}^{\beta} .
$$

Collecting these facts, it follows that

$$
\left|D_{\left(0, h^{(5)}\left(y_{\lambda}\right)\right)}\right| \leq\left\{1-h^{(5)}\left(y_{\lambda}\right)\right\} f^{-1}\left(h^{(5)}\left(y_{\lambda}\right)\right)=O\left(\lambda^{-1} \log \lambda\right) .
$$

However, $N\left(C_{\alpha}\right) \subset D_{\left(0, h^{(5)}\left(y_{\lambda}\right)\right)}$ for all $\alpha \in K_{\lambda}:=D_{\lambda}^{*} \cap\left\{\left[0, x_{\lambda}\right] \times[0,1]\right\}$, implying that

$$
\Xi\left(N\left(C_{\alpha}\right)\right) \leq H\left(D_{\left(0, h^{(5)}\left(y_{\lambda}\right)\right)}\right) .
$$

It thus follows easily that

$$
\int_{K_{\lambda}} \mu_{2}(\mathrm{~d} \alpha) \mathrm{E} \Xi^{2}\left(N\left(C_{\alpha}\right)\right) \text { and } \int_{K_{\lambda}} \mu_{2}(\mathrm{~d} \alpha) \mathrm{E}^{\alpha} \Xi^{2}\left(N\left(C_{\alpha}\right)\right)
$$

are both of order

$$
O\left(\lambda\left|D_{\left(0, h^{(5)}\left(y_{\lambda}\right)\right)}\right| \log ^{2} \lambda\right)=O\left(\log ^{3} \lambda\right)=O\left(\lambda^{1 / 2} \log \lambda\right),
$$

and that

$$
\sup _{\alpha \in K_{\lambda}} \mu_{2}\left(N\left(C_{\alpha}\right)\right)=O(\log \lambda) .
$$

Thus, $\varepsilon_{3}^{\prime}, \varepsilon_{4}^{\prime}$, and $\varepsilon_{5}^{\prime}$ are still of order $O\left(\lambda^{-1 / 4} \log \lambda\right)$ under these less restrictive conditions on $f$.

Note that the same approach could have been used to treat more complicated functions of the process of maximal points; for instance the sum, $\int_{D} D_{\alpha} \Xi(\mathrm{d} \alpha)$, of the areas in $D$ which are above and to the right of maximal points.

\section{The proofs}

We use Theorem 2.1 of Chen and Shao (2004), a discrete version of our Theorem 2.1, to prove Theorem 2.1 , by means of a direct dissection argument.

For each $n$, recalling condition (LD1), the family of open sets $\left\{B^{\circ}\left(\alpha, r_{n}\right), \alpha \in \Gamma\right\}$, where $B^{\circ}(\alpha, r)=\{y: d(y, \alpha)<r\}$, is a covering of $\Gamma$, so it contains a finite subcovering, $\left\{B^{\circ}\left(\alpha_{n i}, r_{n}\right)\right.$, $\left.i=1,2, \ldots, k_{n}^{\prime}\right\}$, of $\Gamma$. Let $B_{n 1}^{\prime}=B^{\circ}\left(\alpha_{n 1}, r_{n}\right)$ and $B_{n i}^{\prime}=B^{\circ}\left(\alpha_{n i}, r_{n}\right) \backslash\left(\bigcup_{j=1}^{i-1} B_{n j}^{\prime}\right)$ for $2 \leq i \leq k_{n}^{\prime}$. Now, for each $n \geq 1$, list all the sets

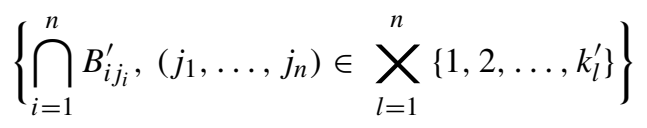

as $\left\{B_{n 1}, \ldots, B_{n k_{n}}\right\}$; then $\left\{\left\{B_{n 1}, \ldots, B_{n k_{n}}\right\}, n \geq 1\right\}$ forms a dissecting system of $\Gamma$ (Daley and Vere-Jones (1988, p. 608)). 
Since $r_{n} \downarrow 0$ as $n \rightarrow \infty$, we can define a nondecreasing sequence of integers $g(n)$ such that $r_{g(n)} \geq 2 r_{n}$ and $\lim _{n \rightarrow \infty} g(n)=\infty$. Define $J_{n i}:=\left\{j: B_{n j} \cap\left(\bigcup_{\alpha \in B_{n i}} A_{\alpha, g(n)}\right) \neq \varnothing\right\}$; then set

$$
M_{n i}=\bigcup_{j \in J_{n i}} B_{n j}
$$

noting that

$$
M_{n i} \supset A_{\alpha, g(n)} \quad \text { for all } \alpha \in B_{n i} .
$$

Lemma 4.1. For each $\alpha \in \Gamma$ and $n \geq 1$, let $j_{n}(\alpha)$ be the value of $j$ such that $\alpha \in B_{n j}$. Then $\alpha \in A_{\alpha} \subset M_{n j_{n}(\alpha)}$ and $M_{n j_{n}(\alpha)} \downarrow A_{\alpha}$ as $n \rightarrow \infty$. Furthermore, defining $J_{n i}^{*}:=$ $\left\{j: B_{n j} \cap M_{n i} \neq \varnothing\right\}$ and $N_{n i}:=\bigcup_{j \in J_{n i}^{*}} B_{n j}$, it also follows that $N_{n j_{n}(\alpha)} \rightarrow A_{\alpha}$ as $n \rightarrow \infty$.

Proof. The first part is clear from the definition of $M_{n i}$ and because $A_{\alpha} \subset A_{\alpha, n}$, so it suffices to prove the last two claims. Note also, from the properties of dissecting systems, that for each $\alpha$ the sets $M_{n j_{n}(\alpha)}$ are decreasing in $n$.

For each $m \geq 1$, let $n_{0}(m)$ be such that $2 r_{n}+r_{g(n)}<r_{m}$ for all $n \geq n_{0}(m)$. Then, for such $n$, it follows that $\left\{y: d\left(y, B_{n j_{n}(\alpha)}\right) \leq r_{g(n)}\right\} \subset B\left(\alpha, r_{m}\right)$, whence $A_{\beta, g(n)} \subset A_{\alpha, m}$ for all $\beta \in B_{n j_{n}(\alpha)}$, by condition (LD1)(b). This implies that

$$
\bigcup_{\beta \in B_{n j_{n}(\alpha)}} A_{\beta, g(n)} \subset A_{\alpha, m}, \quad n \geq n_{0}(m),
$$

and, so, using (4.1), that

$$
A_{\alpha} \subset A_{\alpha, g(n)} \subset M_{n j_{n}(\alpha)} \subset A_{\alpha, m, n}^{(1)}:=\left\{y: d\left(y, A_{\alpha, m}\right)<2 r_{n}\right\}, \quad n \geq n_{0}(m) .
$$

Hence,

$$
A_{\alpha} \subset \bigcap_{n \geq n_{0}(m)} M_{n j_{n}(\alpha)} \subset A_{\alpha, m}
$$

Since, in addition, $A_{\alpha, m} \downarrow A_{\alpha}$ as $m \rightarrow \infty$, by condition (LD1)(a), it follows that $M_{n j_{n}(\alpha)} \downarrow A_{\alpha}$.

To prove the last part, arguing much as above we have

$$
A_{\alpha} \subset N_{n j_{n}(\alpha)} \subset A_{\alpha, m, n}^{(2)}:=\left\{y: d\left(y, A_{\alpha, m}\right)<4 r_{n}\right\}, \quad n \geq n_{0}(m),
$$

and from this the convergence of $N_{n j_{n}(\alpha)}$ to $A_{\alpha}$ follows.

Now, for $1 \leq i \leq k_{n}$, set $X_{n i}:=\tilde{H}_{2}\left(B_{n i}\right)$. Note that, for each $i$ and for any $\beta_{n i} \in B_{n i}$, we have

$$
B_{n i} \subset B\left(\beta_{n i}, 2 r_{n}\right) \subset B\left(\beta_{n i}, r_{g(n)}\right), \quad A_{n i}^{\prime}:=\bigcup_{j \notin J_{n i}^{*}} B_{n j} \subset M_{n i}^{\mathrm{c}}, \quad M_{n i} \supset A_{\beta_{n i}, g(n)},
$$

this last by (4.1). Hence, $X_{n i}$ is a function of $\left.H\right|_{B\left(\beta_{n i}, r_{g(n)}\right)}$, whereas $X_{n j}, j \notin J_{n i}^{*}$, are functions of $\left.H\right|_{A_{n i}^{\prime}}$ and, thus, of $\left.H\right|_{A_{\beta_{n i}, g(n)}^{\mathrm{c}}}$. From condition (LD1)(a), it now follows that $X_{n i}$ is independent of $\left\{X_{n j}, j \notin J_{n i}^{*}\right\}$. We have thus, for each $n$, constructed a discrete collection of random variables, $\left\{X_{n i}, 1 \leq i \leq k_{n}\right\}$, satisfying condition (LD1) of Chen and Shao (2004), in such a way that $\sum_{i=1}^{k_{n}} X_{n i}=\tilde{H}_{2}(\Gamma)$ for all $n$. Hence, in order to prove our Theorem 2.1, we merely need to show that the bound given in Theorem 2.1 of Chen and Shao (2004), with $X_{n i}$ as above and with $Y_{n i}=\sum_{j \in J_{n i}^{*}} X_{n j}$, is itself bounded in the limit as $n \rightarrow \infty$ by the one that we give. This follows from the next lemma. 
Lemma 4.2. Let $f_{1}$ and $f_{2}$ be two nonnegative, continuous functions defined on $\mathbb{R}^{2}$ such that $f_{1}(x, y) \leq|x|+|y|$ and $f_{2}$ is bounded. Under condition (2.1), as $n \rightarrow \infty$ we have

$$
\begin{gathered}
\mathrm{E}\left|\sum_{i=1}^{k_{n}} Y_{n i} X_{n i}-\int_{\Gamma} Y_{\alpha} \tilde{H}_{2}(\mathrm{~d} \alpha)\right| \rightarrow 0 \\
\limsup _{n \rightarrow \infty} \mathrm{E} \sum_{i=1}^{k_{n}} f_{1}\left(\tilde{H}_{2}(\Gamma), Y_{n i}\right) \mathbf{1}_{\left\{\left|Y_{n i}\right|>c\right\}}\left|X_{n i}\right| \\
\leq \mathrm{E} \int_{\Gamma} f_{1}\left(\tilde{H}_{2}(\Gamma), Y_{\alpha}\right) \mathbf{1}_{\left\{\left|Y_{\alpha}\right| \geq c\right\}}\left|\tilde{H}_{2}(\mathrm{~d} \alpha)\right|, \quad c \in \mathbb{R} \\
\mathrm{E} \sum_{i, j=1}^{k_{n}} f_{2}\left(Y_{n i}, Y_{n j}\right) X_{n i} X_{n j} \rightarrow \mathrm{E} \iint_{\Gamma^{2}} f_{2}\left(Y_{\alpha}, Y_{\beta}\right) \tilde{H}_{2}(\mathrm{~d} \alpha) \tilde{H}_{2}(\mathrm{~d} \beta), \\
\mathrm{E} \sum_{i, j=1}^{k_{n}} f_{2}\left(Y_{n i}, Y_{n j}^{*}\right) X_{n i} X_{n j}^{*} \rightarrow \mathrm{E} \iint_{\Gamma^{2}} f_{2}\left(Y_{\alpha}, Y_{\beta}\right) \tilde{H}_{2}(\mathrm{~d} \alpha) \tilde{H}_{2}^{*}(\mathrm{~d} \beta),
\end{gathered}
$$

where $\left\{X_{n j}^{*}, Y_{n j}^{*}, 1 \leq j \leq k_{n}\right\}$ is an independent copy of $\left\{X_{n j}, Y_{n j}, 1 \leq j \leq k_{n}\right\}$.

Proof. We prove (4.3) and (4.4); the proof of the other two claims can be accomplished in the same way as the proof of (4.4). To prove (4.3), note that

$$
\begin{aligned}
& \mathrm{E} \sum_{i=1}^{k_{n}} f_{1}\left(\tilde{H}_{2}(\Gamma), Y_{n i}\right) \mathbf{1}_{\left\{\left|Y_{n i}\right|>c\right\}}\left|X_{n i}\right|-\mathrm{E} \int_{\Gamma} f_{1}\left(\tilde{H}_{2}(\Gamma), Y_{\alpha}\right) \mathbf{1}_{\left\{\left|Y_{\alpha}\right| \geq c\right\}}\left|\tilde{H}_{2}(\mathrm{~d} \alpha)\right| \\
& =\mathrm{E} \sum_{i=1}^{k_{n}} f_{1}\left(\tilde{H}_{2}(\Gamma), Y_{n i}\right) \mathbf{1}_{\left\{\left|Y_{n i}\right|>c\right\}}\left\{\left|X_{n i}\right|-\int_{B_{n i}}\left|\tilde{H}_{2}(\mathrm{~d} \alpha)\right|\right\} \\
& \quad+\mathrm{E} \sum_{i=1}^{k_{n}} \int_{B_{n i}}\left[f_{1}\left(\tilde{H}_{2}(\Gamma), Y_{n i}\right) \mathbf{1}_{\left\{\left|Y_{n i}\right|>c\right\}}-f_{1}\left(\tilde{H}_{2}(\Gamma), Y_{\alpha}\right) \mathbf{1}_{\left\{\left|Y_{\alpha}\right| \geq c\right\}}\right]\left|\tilde{H}_{2}(\mathrm{~d} \alpha)\right| .
\end{aligned}
$$

The quantity (4.6) is clearly nonpositive. In (4.7), the first element is bounded above by

$$
\mathrm{E} \int_{\Gamma} \sup _{m \geq n}\left\{f_{1}\left(\tilde{H}_{2}(\Gamma), Y_{m j_{m}(\alpha)}\right) \mathbf{1}_{\left\{\left|Y_{m j_{m}(\alpha)}\right|>c\right\}}\right\}\left|\tilde{H}_{2}(\mathrm{~d} \alpha)\right|,
$$

which, as $n \rightarrow \infty$, converges to

$$
\mathrm{E} \int_{\Gamma} \limsup _{n \rightarrow \infty}\left\{f_{1}\left(\tilde{H}_{2}(\Gamma), Y_{n j_{n}(\alpha)}\right) \mathbf{1}_{\left\{\left|Y_{n j_{n}(\alpha)}\right|>c\right\}}\right\}\left|\tilde{H}_{2}(\mathrm{~d} \alpha)\right|,
$$

by monotone convergence. Now, by Lemma 4.1 we have $N_{n j_{n}(\alpha)} \rightarrow A_{\alpha}$ and, hence, $Y_{n j_{n}(\alpha)} \rightarrow$ $Y_{\alpha}$; thus, the integrand in (4.8) is just

$$
f_{1}\left(\tilde{H}_{2}(\Gamma), Y_{\alpha}\right) \limsup _{n \rightarrow \infty}\left\{\mathbf{1}_{\left\{\left|Y_{n j_{n}(\alpha)}\right|>c\right\}}\right\} \leq f_{1}\left(\tilde{H}_{2}(\Gamma), Y_{\alpha}\right) \mathbf{1}_{\left\{\left|Y_{\alpha}\right| \geq c\right\}},
$$

implying that the limit supremum of (4.7) is also nonpositive. 
To prove (4.4), note that

$$
\begin{aligned}
& \left|\mathrm{E} \sum_{i, j=1}^{k_{n}} f_{2}\left(Y_{n i}, Y_{n j}\right) X_{n i} X_{n j}-\mathrm{E} \iint_{\Gamma^{2}} f_{2}\left(Y_{\alpha}, Y_{\beta}\right) \tilde{H}_{2}(\mathrm{~d} \alpha) \tilde{H}_{2}(\mathrm{~d} \beta)\right| \\
& \quad \leq \mathrm{E} \sum_{i, j=1}^{k_{n}} \int_{B_{n i}} \int_{B_{n j}}\left|f_{2}\left(Y_{n i}, Y_{n j}\right)-f_{2}\left(Y_{\alpha}, Y_{\beta}\right)\right|\left|\tilde{H}_{2}(\mathrm{~d} \alpha) \tilde{H}_{2}(\mathrm{~d} \beta)\right| .
\end{aligned}
$$

In view of (2.1), dominated convergence completes the proof.

Proof of Theorem 2.1. Using Theorem 2.1 of Chen and Shao (2004), we have

$$
d_{\mathrm{K}}\left(\mathcal{L}\left(\vartheta^{-1}(W-\mathrm{E} W)\right), \mathcal{N}(0,1)\right) \leq r_{1}^{n}+4 r_{2}^{n}+8 r_{3}^{n}+r_{4}^{n}+4.5 r_{5}^{n}+1.5 r_{6}^{n},
$$

for all $n$, where

$$
\begin{aligned}
& r_{1}^{n}=\mathrm{E}\left|\sum_{i=1}^{k_{n}}\left(X_{n i} Y_{n i}-\mathrm{E} X_{n i} Y_{n i}\right)\right| \\
& r_{2}^{n}=\sum_{i=1}^{k_{n}} \mathrm{E}\left|X_{n i} Y_{n i}\right| \mathbf{1}_{\left\{\left|Y_{n i}\right|>1\right\}}, \\
& r_{3}^{n}= \sum_{i=1}^{k_{n}} \mathrm{E}\left|X_{n i}\right|\left(Y_{n i}^{2} \wedge 1\right), \\
& r_{4}^{n}= \sum_{i=1}^{k_{n}} \mathrm{E}\left\{\left|\tilde{H}_{2}(\Gamma) X_{n i}\right|\left(Y_{n i}^{2} \wedge 1\right)\right\} \\
& r_{5}^{n}= \sum_{i, j=1}^{k_{n}} \mathrm{E}\left\{X_{n i} X_{n j} \mathbf{1}_{\left\{Y_{n i} Y_{n j} \geq 0\right\}}\left(\left|Y_{n i}\right| \wedge\left|Y_{n j}\right| \wedge 1\right)\right. \\
&\left(r_{6}^{n}\right)^{2}= \frac{1}{2} \sum_{i, j=1}^{k_{n}} \mathrm{E}\left\{X_{n i} X_{n j} \mathbf{1}_{\left\{Y_{n i} Y_{n j} \geq 0\right\}}\left(\left|Y_{n i}\right|^{2} \wedge\left|Y_{n j}\right|^{2} \wedge 1\right)\right. \\
&\left.\quad-X_{n i} X_{n j}^{*} \mathbf{1}_{\left\{Y_{n i} Y_{n j}^{*} \geq 0\right\}}\left(\left|Y_{n i}\right|^{2} \wedge\left|Y_{n j}^{*}\right|^{2} \wedge 1\right)\right\}
\end{aligned}
$$

Using Lemma 4.2, we have $r_{1}^{n} \rightarrow r_{1}$ by (4.2); $\lim _{\sup } \rightarrow \infty r_{l}^{n} \leq r_{l}, l=2,3$, 4, by (4.3) with $c=1,-1,-1$, respectively; and $r_{5}^{n} \rightarrow r_{5}$ and $r_{6}^{n} \rightarrow r_{6}$ by (4.4) and (4.5), respectively. Finally, direct calculation yields

$$
r_{5}=\int_{|t| \leq 1} \operatorname{var} \hat{K}(t) \mathrm{d} t, \quad\left(r_{6}\right)^{2}=\int_{|t| \leq 1}|t| \operatorname{var} \hat{K}(t) \mathrm{d} t .
$$

Proof of Theorem 2.2. Recalling that $p_{3}=p \wedge 3$, for $p \geq 2$ we immediately have

$$
r_{2} \leq \mathrm{E} \int_{\Gamma}\left|Y_{\alpha}\right|^{p_{3}-1}\left|\tilde{H}_{2}(\mathrm{~d} \alpha)\right|=\tilde{r}_{1}\left(p_{3}\right)
$$


and

$$
r_{3}=\mathrm{E} \int_{\Gamma}\left\{Y_{\alpha}^{2} \wedge 1\right\}\left|\tilde{H}_{2}(\mathrm{~d} \alpha)\right| \leq \mathrm{E} \int_{\Gamma}\left|Y_{\alpha}\right|^{p_{3}-1}\left|\tilde{H}_{2}(\mathrm{~d} \alpha)\right|=\tilde{r}_{1}\left(p_{3}\right) .
$$

For $r_{5}$, using the independence of $Y_{\alpha}$ and $Y_{\beta}$ when $(\alpha, \beta) \notin B^{*}$, we obtain

$$
\begin{aligned}
r_{5} & \leq \mathrm{E} \iint_{B^{*}}\left|Y_{\alpha}\right|^{p_{3}-2}\left|\tilde{H}_{2}(\mathrm{~d} \alpha)\right|\left|\tilde{H}_{2}(\mathrm{~d} \beta)\right|+\mathrm{E} \iint_{B^{*}}\left|Y_{\alpha}\right|^{p_{3}-2}\left|\tilde{H}_{2}(\mathrm{~d} \alpha)\right|\left|\tilde{H}_{2}^{*}(\mathrm{~d} \beta)\right| \\
& =\tilde{r}_{2}\left(p_{3}\right),
\end{aligned}
$$

and, similarly, using the same argument but with $p_{3}$ replaced by $p$, we have

$$
r_{6}^{2} \leq \frac{1}{2} \mathrm{E} \iint_{B^{*}}\left|Y_{\alpha}\right|^{p-2}\left[\left|\tilde{H}_{2}(\mathrm{~d} \alpha)\right|\left|\tilde{H}_{2}(\mathrm{~d} \beta)\right|+\left|\tilde{H}_{2}(\mathrm{~d} \alpha)\right|\left|\tilde{H}_{2}^{*}(\mathrm{~d} \beta)\right|\right]=\frac{1}{2} \tilde{r}_{2}(p) .
$$

To find $r_{4}$, recalling the notation $\tilde{W}=\tilde{H}_{2}(\Gamma)$ of Section 2, we note that

$$
\mathrm{E}\left|\tilde{W}-Z_{\alpha}\right| \leq \mathrm{E}|\tilde{W}|+\mathrm{E}\left|Z_{\alpha}\right| \leq \sqrt{\operatorname{var}(\tilde{W})}+\mathrm{E}\left|Z_{\alpha}\right| \leq 1+\mathrm{E} \int_{\beta \in B_{\alpha}}\left|\tilde{H}_{2}(\mathrm{~d} \beta)\right|
$$

and that $\tilde{W}-Z_{\alpha}$ is independent of $\left.\tilde{H}_{2}\right|_{A_{\alpha}}$; hence, it follows that

$$
\begin{aligned}
r_{4}= & \mathrm{E}\left\{|\tilde{W}| \int_{\Gamma}\left\{Y_{\alpha}^{2} \wedge 1\right\}\left|\tilde{H}_{2}(\mathrm{~d} \alpha)\right|\right\} \\
\leq & \mathrm{E} \int_{\Gamma}\left\{\left|\tilde{W}-Z_{\alpha}\right|+\left|Z_{\alpha}\right|\right\}\left\{Y_{\alpha}^{2} \wedge 1\right\}\left|\tilde{H}_{2}(\mathrm{~d} \alpha)\right| \\
\leq & \mathrm{E} \int_{\Gamma}\left[1+\mathrm{E} \int_{B_{\alpha}}\left|\tilde{H}_{2}(\mathrm{~d} \beta)\right|\right]\left\{Y_{\alpha}^{2} \wedge 1\right\}\left|\tilde{H}_{2}(\mathrm{~d} \alpha)\right| \\
& +\mathrm{E} \int_{\Gamma}\left\{Y_{\alpha}^{2} \wedge 1\right\}\left[\int_{B_{\alpha}}\left|\tilde{H}_{2}(\mathrm{~d} \beta)\right|\right]\left|\tilde{H}_{2}(\mathrm{~d} \alpha)\right| \\
\leq & \mathrm{E} \int_{\Gamma}\left\{\left|Y_{\alpha}\right|^{p_{3}-1}+\left|Y_{\alpha}\right|^{p_{3}-2} \int_{B_{\alpha}}\left(\left|\tilde{H}_{2}^{*}(\mathrm{~d} \beta)\right|+\left|\tilde{H}_{2}(\mathrm{~d} \beta)\right|\right)\right\}\left|\tilde{H}_{2}(\mathrm{~d} \alpha)\right| \\
\leq & \tilde{r}_{1}\left(p_{3}\right)+\tilde{r}_{2}\left(p_{3}\right) .
\end{aligned}
$$

Finally,

$$
r_{1} \leq \mathrm{E}\left|\int_{\Gamma} Y_{\alpha} \mathbf{1}_{\left\{\left|Y_{\alpha}\right| \leq 1\right\}} \tilde{H}_{2}(\mathrm{~d} \alpha)-\mathrm{E} \int_{\Gamma} Y_{\alpha} \mathbf{1}_{\left\{\left|Y_{\alpha}\right| \leq 1\right\}} \tilde{H}_{2}(\mathrm{~d} \alpha)\right|+2 r_{2}=: r_{1}^{\prime}+2 r_{2},
$$

where, temporarily writing $h_{1}(y):=y \mathbf{1}_{[-1,1]}(y)$,

$$
\begin{aligned}
\left(r_{1}^{\prime}\right)^{2} & \leq \operatorname{var} \int_{\Gamma} Y_{\alpha} \mathbf{1}_{\left\{\left|Y_{\alpha}\right| \leq 1\right\}} \tilde{H}_{2}(\mathrm{~d} \alpha) \\
& =\mathrm{E} \iint_{\Gamma^{2}}\left\{h_{1}\left(Y_{\alpha}\right) h_{1}\left(Y_{\beta}\right) \tilde{H}_{2}(\mathrm{~d} \alpha) \tilde{H}_{2}(\mathrm{~d} \beta)-h_{1}\left(Y_{\alpha}\right) h_{1}\left(Y_{\beta}^{*}\right) \tilde{H}_{2}(\mathrm{~d} \alpha) \tilde{H}_{2}^{*}(\mathrm{~d} \beta)\right\} \\
& \leq \mathrm{E} \iint_{B^{*}}\left\{\left|h_{1}\left(Y_{\alpha}\right) h_{1}\left(Y_{\beta}\right) \tilde{H}_{2}(\mathrm{~d} \alpha) \tilde{H}_{2}(\mathrm{~d} \beta)\right|+\left|h_{1}\left(Y_{\alpha}\right) h_{1}\left(Y_{\beta}^{*}\right) \tilde{H}_{2}(\mathrm{~d} \alpha) \tilde{H}_{2}^{*}(\mathrm{~d} \beta)\right|\right\} .
\end{aligned}
$$


Since $\left|y_{1} y_{2}\right| \leq \frac{1}{2}\left(y_{1}^{2}+y_{2}^{2}\right)$, it follows that

$$
\begin{aligned}
\left(r_{1}^{\prime}\right)^{2} & \leq \mathrm{E} \iint_{B^{*}} Y_{\alpha}^{2} \mathbf{1}_{\left\{\left|Y_{\alpha}\right| \leq 1\right\}}\left|\tilde{H}_{2}(\mathrm{~d} \alpha)\right|\left(\left|\tilde{H}_{2}(\mathrm{~d} \beta)\right|+\left|\tilde{H}_{2}^{*}(\mathrm{~d} \beta)\right|\right) \\
& \leq \mathrm{E} \iint_{B^{*}}\left|Y_{\alpha}\right|^{p-2}\left|\tilde{H}_{2}(\mathrm{~d} \alpha)\right|\left(\left|\tilde{H}_{2}(\mathrm{~d} \beta)\right|+\left|\tilde{H}_{2}^{*}(\mathrm{~d} \beta)\right|\right) \\
& =\tilde{r}_{2}(p) .
\end{aligned}
$$

Collecting the estimates for $r_{i}, i=1, \ldots, 6$, and substituting them into the bound in Theorem 2.1 gives the result.

To prove Theorem 2.3, we need the following result, which is similar to, but slightly different from, Proposition 3.2 of Chen and Shao (2004). Although the proof follows rather directly from theirs, we prefer to give it here for the sake of completeness.

Proposition 4.1. Assume that condition (LD3) holds, and let $\eta(\alpha):=\left.\tilde{H}_{2}\right|_{B_{\alpha}}$. Then, for any $a \equiv a(\eta(\alpha))$ and $b \equiv b(\eta(\alpha))$, we have

$$
\begin{aligned}
\mathrm{P}^{\eta(\alpha)}(a \leq \tilde{W} \leq b) & \leq \frac{1}{8}\left(4 u_{\alpha}+5\right)(b-a)+\frac{1}{8}\left(12 u_{\alpha}+17\right) r_{3}+4 r_{2}+2 r_{2, \alpha}^{\prime}+4 r_{10} \\
& \leq \frac{1}{8}\left(4 u_{\alpha}+5\right)(b-a)+\frac{1}{8}\left(12 u_{\alpha}+17\right) r_{3}+4 r_{2}+2 r_{14}+4 r_{10},
\end{aligned}
$$

where $\mathrm{P}^{\eta(\alpha)}$ denotes probability conditional on the $\sigma$-field generated by $\eta(\alpha)$,

$$
u_{\alpha}=\mathrm{E}\left|\tilde{H}_{2}\left(N\left(C_{\alpha}\right)\right)\right|, \quad \text { and } r_{2, \alpha}^{\prime}=\left|\mathrm{E} \int_{N\left(C_{\alpha}\right)}\left[\left((-1) \vee Y_{\beta}\right) \wedge 1\right] \tilde{H}_{2}(\mathrm{~d} \beta)\right| \text {. }
$$

Proof. Let $f_{\eta(\alpha)}$ be defined by fixing $f_{\eta(\alpha)}((a+b) / 2)=0$ and setting $f_{\eta(\alpha)}^{\prime}$ to be the continuous function given by

$$
f_{\eta(\alpha)}^{\prime}(w)= \begin{cases}1 & \text { for } a \leq w \leq b, \\ 0 & \text { for } w \leq a-r_{3} \text { and } w \geq b+r_{3}, \\ \left(w-a+r_{3}\right) / r_{3} & \text { for } a-r_{3}<w<a, \\ \left(b+r_{3}-w\right) / r_{3} & \text { for } b<w<b+r_{3} .\end{cases}
$$

Then $\left|f_{\eta(\alpha)}(w)\right| \leq\left(b-a+r_{3}\right) / 2$. Let

$$
\hat{M}(t):=\int_{N\left(C_{\alpha}\right)^{\mathrm{c}}} \hat{K}(t, \mathrm{~d} \beta), \quad M(t):=\mathrm{E} \hat{M}(t),
$$

let $\mathrm{E}^{\eta(\alpha)}$ stand for the conditional expectation in terms of the $\sigma$-field generated by $\eta(\alpha)$, and let $\tilde{W}_{\alpha}:=\int_{N\left(C_{\alpha}\right)^{\mathrm{c}}} \tilde{H}_{2}(\mathrm{~d} \beta)$. Owing to the independence between $\left.\tilde{H}_{2}\right|_{B_{\alpha}}$ and $\left.\tilde{H}_{2}\right|_{N\left(C_{\alpha}\right)^{\mathrm{c}}}$, we have

$$
\begin{aligned}
\frac{1}{2}(b- & \left.a+r_{3}\right)\left(1+u_{\alpha}\right) \geq \frac{1}{2}\left(b-a+r_{3}\right) \mathrm{E}\left|\tilde{H}_{2}\left(N\left(C_{\alpha}\right)^{\mathrm{c}}\right)\right| \\
& \geq \mathrm{E}^{\eta(\alpha)}\left\{\tilde{H}_{2}\left(N\left(C_{\alpha}\right)^{\mathrm{c}}\right) f_{\eta(\alpha)}(\tilde{W})\right\} \\
& =\mathrm{E}^{\eta(\alpha)} \int_{N\left(C_{\alpha}\right)^{\mathrm{c}}}\left(f_{\eta(\alpha)}(\tilde{W})-f_{\eta(\alpha)}\left(\tilde{W}-Y_{\beta}\right)\right) \tilde{H}_{2}(\mathrm{~d} \beta) \\
& =\mathrm{E}^{\eta(\alpha)} \int_{-\infty}^{\infty} f_{\eta(\alpha)}^{\prime}(\tilde{W}+t) \hat{M}(t) \mathrm{d} t \\
& =: \sum_{i=1}^{4} H_{i, \eta(\alpha)},
\end{aligned}
$$


where

$$
\begin{aligned}
& H_{1, \eta(\alpha)}=\mathrm{E}^{\eta(\alpha)}\left\{f_{\eta(\alpha)}^{\prime}(\tilde{W}) \int_{|t| \leq 1} M(t) \mathrm{d} t\right\} \\
& H_{2, \eta(\alpha)}=\mathrm{E}^{\eta(\alpha)} \int_{|t| \leq 1}\left(f_{\eta(\alpha)}^{\prime}(\tilde{W}+t)-f_{\eta(\alpha)}^{\prime}(\tilde{W})\right) M(t) \mathrm{d} t, \\
& H_{3, \eta(\alpha)}=\mathrm{E}^{\eta(\alpha)} \int_{|t|>1} f_{\eta(\alpha)}^{\prime}(\tilde{W}+t) \hat{M}(t) \mathrm{d} t \\
& H_{4, \eta(\alpha)}=\mathrm{E}^{\eta(\alpha)} \int_{|t| \leq 1} f_{\eta(\alpha)}^{\prime}(\tilde{W}+t)(\hat{M}(t)-M(t)) \mathrm{d} t .
\end{aligned}
$$

Noting that $\eta(\alpha)$ and $\hat{M}(t)$ are independent and that

$$
1=\mathrm{E}\left(\tilde{W}^{2}\right)=\mathrm{E} \int_{\Gamma} Y_{\beta} \tilde{H}_{2}(\mathrm{~d} \beta)
$$

we obtain

$$
\begin{aligned}
\int_{|t| \leq 1} M(t) \mathrm{d} t= & \mathrm{E} \int_{N\left(C_{\alpha}\right)^{\mathrm{c}}}\left[\left((-1) \vee Y_{\beta}\right) \wedge 1\right] \tilde{H}_{2}(\mathrm{~d} \beta) \\
= & 1-\mathrm{E} \int_{\Gamma}\left\{Y_{\beta}-\left[\left((-1) \vee Y_{\beta}\right) \wedge 1\right]\right\} \tilde{H}_{2}(\mathrm{~d} \beta) \\
& -\mathrm{E} \int_{N\left(C_{\alpha}\right)}\left[\left((-1) \vee Y_{\beta}\right) \wedge 1\right] \tilde{H}_{2}(\mathrm{~d} \beta) \\
\geq & 1-\mathrm{E} \int_{\Gamma}\left|Y_{\beta}\right| \mathbf{1}_{\left\{\left|Y_{\beta}\right|>1\right\}}\left|\tilde{H}_{2}(\mathrm{~d} \beta)\right|-\mathrm{E} \int_{N\left(C_{\alpha}\right)}\left[\left((-1) \vee Y_{\beta}\right) \wedge 1\right] \tilde{H}_{2}(\mathrm{~d} \beta) \\
\geq & 1-r_{2}-r_{2, \alpha}^{\prime}
\end{aligned}
$$

and, hence,

$$
H_{1, \eta(\alpha)} \geq \mathrm{E}^{\eta(\alpha)} f_{\eta(\alpha)}^{\prime}(\tilde{W})\left(1-r_{2}-r_{2, \alpha}^{\prime}\right) \geq \mathrm{P}^{\eta(\alpha)}(a \leq \tilde{W} \leq b)-r_{2}-r_{2, \alpha}^{\prime} .
$$

Also,

$$
\left|H_{3, \eta(\alpha)}\right| \leq \mathrm{E}^{\eta(\alpha)} \int_{N\left(C_{\alpha}\right)^{\mathrm{c}}}\left|Y_{\beta}\right| \mathbf{1}_{\left\{\left|Y_{\beta}\right|>1\right\}}\left|\tilde{H}_{2}(\mathrm{~d} \beta)\right|=\mathrm{E} \int_{N\left(C_{\alpha}\right)^{\mathrm{c}}}\left|Y_{\beta}\right| \mathbf{1}_{\left\{\left|Y_{\beta}\right|>1\right\}}\left|\tilde{H}_{2}(\mathrm{~d} \beta)\right| \leq r_{2}
$$

and

$$
\begin{aligned}
\left|H_{4, \eta(\alpha)}\right| & \leq \frac{1}{8} \mathrm{E}^{\eta(\alpha)} \int_{|t| \leq 1}\left[f_{\eta(\alpha)}^{\prime}(\tilde{W}+t)\right]^{2} \mathrm{~d} t+2 \mathrm{E}^{\eta(\alpha)} \int_{|t| \leq 1}(\hat{M}(t)-M(t))^{2} \mathrm{~d} t \\
& \leq \frac{1}{8}\left(b-a+2 r_{3}\right)+2 \rho,
\end{aligned}
$$


where, temporarily writing $h(y, t):=\mathbf{1}_{[-y, 0)}(t)-\mathbf{1}_{[0,-y]}(t)$,

$$
\begin{aligned}
\rho & =\mathrm{E}^{\eta(\alpha)} \int_{|t| \leq 1}(\hat{M}(t)-M(t))^{2} \mathrm{~d} t=\mathrm{E} \int_{|t| \leq 1}(\hat{M}(t)-M(t))^{2} \mathrm{~d} t \\
& =\mathrm{E} \iint_{\left[N\left(C_{\alpha}\right)^{\mathrm{c}}\right]^{2}} \int_{|t| \leq 1}\left\{h\left(Y_{\beta_{1}}, t\right) h\left(Y_{\beta_{2}}, t\right) \tilde{H}_{2}\left(\mathrm{~d} \beta_{1}\right) \tilde{H}_{2}\left(\mathrm{~d} \beta_{2}\right)\right. \\
& \left.-h\left(Y_{\beta_{1}}, t\right) h\left(Y_{\beta_{2}}^{*}, t\right) \tilde{H}_{2}\left(\mathrm{~d} \beta_{1}\right) \tilde{H}_{2}^{*}\left(\mathrm{~d} \beta_{2}\right)\right\}
\end{aligned}
$$

To bound $H_{2, \eta(\alpha)}$, define

$$
L_{\eta(\alpha)}(c)=\lim _{k \rightarrow \infty} \sup _{x \in \mathbb{Q}} \mathrm{P}^{\eta(\alpha)}\left(x-\frac{1}{k} \leq \tilde{W} \leq x+\frac{1}{k}+c\right),
$$

where $\mathbb{Q}$ is the set of all rational numbers. Since

$$
\begin{aligned}
\left|\mathrm{E}^{\eta(\alpha)}\left\{f_{\eta(\alpha)}^{\prime}(\tilde{W}+t)-f_{\eta(\alpha)}^{\prime}(\tilde{W})\right\}\right| & =\left|\int_{0}^{t} \mathrm{E}^{\eta(\alpha)} f_{\eta(\alpha)}^{\prime \prime}(\tilde{W}+s) \mathrm{d} s\right| \\
& \leq \frac{1}{r_{3}} L_{\eta(\alpha)}\left(r_{3}\right)\left|\int_{0}^{t} \mathrm{~d} s\right| \\
& =\frac{|t| L_{\eta(\alpha)}\left(r_{3}\right)}{r_{3}},
\end{aligned}
$$

we have

$$
\begin{aligned}
\left|H_{2, \eta(\alpha)}\right| & \leq \frac{L_{\eta(\alpha)}\left(r_{3}\right)}{r_{3}} \int_{|t| \leq 1}|t||M(t)| \mathrm{d} t \leq \frac{L_{\eta(\alpha)}\left(r_{3}\right)}{2 r_{3}} \mathrm{E} \int_{N\left(C_{\alpha}\right)^{\mathrm{c}}}\left\{Y_{\beta}^{2} \wedge 1\right\}\left|\tilde{H}_{2}(\mathrm{~d} \beta)\right| \\
& \leq \frac{1}{2} L_{\eta(\alpha)}\left(r_{3}\right) .
\end{aligned}
$$

Therefore, collecting the estimates of $H_{i, \eta(\alpha)}, i=1,2,3,4$, gives

$$
\mathrm{P}^{\eta(\alpha)}(a \leq \tilde{W} \leq b) \leq \frac{1}{8}\left(4 u_{\alpha}+5\right)(b-a)+\frac{1}{4}\left(2 u_{\alpha}+3\right) r_{3}+2 r_{2}+r_{2, \alpha}^{\prime}+2 r_{10}+\frac{1}{2} L_{\eta(\alpha)}\left(r_{3}\right) .
$$

Setting $a=x-1 / k$ and $b=x+1 / k+r_{3}$, taking the supremum over $x \in \mathbb{Q}$, and then taking the limit in terms of $k \rightarrow \infty$ gives

$$
\frac{1}{2} L_{\eta(\alpha)}\left(r_{3}\right) \leq\left(u_{\alpha}+\frac{11}{8}\right) r_{3}+2 r_{2}+r_{2, \alpha}^{\prime}+2 r_{10},
$$


and combining this with (4.9) yields

$$
\mathrm{P}^{\eta(\alpha)}(a \leq \tilde{W} \leq b) \leq \frac{1}{8}\left(4 u_{\alpha}+5\right)(b-a)+\frac{1}{8}\left(12 u_{\alpha}+17\right) r_{3}+4 r_{2}+2 r_{2, \alpha}^{\prime}+4 r_{10} .
$$

Proof of Theorem 2.3. Let

$$
h_{z, c}(w)= \begin{cases}1 & \text { for } w<z \\ 1+(z-w) / c & \text { for } z \leq w \leq z+c \\ 0 & \text { for } w>z+c\end{cases}
$$

and let $f_{z, c}$ be the solution to the Stein equation

$$
f_{z, c}^{\prime}(w)-w f_{z, c}(w)=h_{z, c}(w)-\Phi\left(h_{z, c}\right),
$$

where $\Phi(h):=(1 / \sqrt{2 \pi}) \int_{-\infty}^{\infty} h(x) \mathrm{e}^{-x^{2} / 2} \mathrm{~d} x$. Then, from Chen and Shao (2004, p. 2010), we have

$$
\begin{gathered}
0 \leq f_{z, c}(w) \leq 1, \quad\left|f_{z, c}^{\prime}(w)\right| \leq 1, \quad\left|f_{z, c}^{\prime}\left(w_{1}\right)-f_{z, c}^{\prime}\left(w_{2}\right)\right| \leq 1 \\
\left|f_{z, c}^{\prime}(w+s)-f_{z, c}^{\prime}(w+t)\right| \leq(|w|+1) \min \{|s|+|t|, 1\}+\frac{1}{c}\left|\int_{s}^{t} \mathbf{1}_{\{z \leq w+u \leq z+c\}} \mathrm{d} u\right| .
\end{gathered}
$$

Writing $F(z):=\mathrm{P}(\tilde{W} \leq z)$, we note that

$$
\sup _{z}|F(z)-\Phi(z)| \leq \frac{1}{5} c+\sup _{z}\left|\mathrm{E} h_{z, c}(\tilde{W})-\Phi\left(h_{z, c}\right)\right| .
$$

For $F(z)<\Phi(z)$, this follows because

$$
\begin{aligned}
|F(z)-\Phi(z)| & =\Phi(z)-F(z) \\
& \leq\left\{\Phi(z)-\Phi\left(h_{z-c, c}\right)\right\}+\left\{\Phi\left(h_{z-c, c}\right)-\mathrm{E} h_{z-c, c}(\tilde{W})\right\} \\
& \leq \frac{c}{2 \sqrt{2 \pi}}+\sup _{z}\left|\mathrm{E} h_{z, c}(\tilde{W})-\Phi\left(h_{z, c}\right)\right| ;
\end{aligned}
$$

the argument for $F(z) \geq \Phi(z)$ is analogous.

Let $K(t, \mathrm{~d} \alpha)=\mathrm{E} \hat{K}(t, \mathrm{~d} \alpha)$. Since $\left.\tilde{\tilde{H}}_{2}\right|_{\{\alpha\}}$ is independent of $\left.\tilde{H}_{2}\right|_{A_{\alpha}^{c}}$ in the sense of condition (LD1)(a) and $\left.\tilde{H}_{2}\right|_{A_{\alpha}}$ is independent of $\tilde{W}-Z_{\alpha}$, we have

$$
\begin{aligned}
\mathrm{E} f_{z, c}^{\prime}(\tilde{W})-\mathrm{E}\left\{\tilde{W} f_{z, c}(\tilde{W})\right\} \\
=\mathrm{E}\left\{\int_{\Gamma} \int_{-\infty}^{\infty} f_{z, c}^{\prime}(\tilde{W}) K(t, \mathrm{~d} \alpha) \mathrm{d} t-\int_{\Gamma} \int_{-\infty}^{\infty} f_{z, c}^{\prime}(\tilde{W}+t) \hat{K}(t, \mathrm{~d} \alpha) \mathrm{d} t\right\} \\
=\mathrm{E} \int_{\Gamma}^{\infty} \int_{-\infty}^{\infty}\left[f_{z, c}^{\prime}(\tilde{W})-f_{z, c}^{\prime}\left(\tilde{W}-Z_{\alpha}+t\right)\right] K(t, \mathrm{~d} \alpha) \mathrm{d} t \\
+\mathrm{E} \int_{\Gamma} \int_{-\infty}^{\infty}\left[f_{z, c}^{\prime}\left(\tilde{W}-Z_{\alpha}+t\right)-f_{z, c}^{\prime}(\tilde{W}+t)\right] \hat{K}(t, \mathrm{~d} \alpha) \mathrm{d} t \\
=Q_{1}+Q_{2}+Q_{3}+Q_{4},
\end{aligned}
$$


where

$$
\begin{aligned}
& Q_{1}:=\mathrm{E} \int_{\Gamma} \int_{|t| \leq 1}\left(f_{z, c}^{\prime}(\tilde{W})-f_{z, c}^{\prime}\left(\tilde{W}-Z_{\alpha}+t\right)\right) K(t, \mathrm{~d} \alpha) \mathrm{d} t, \\
& Q_{2}:=\mathrm{E} \int_{\Gamma} \int_{|t|>1}\left(f_{z, c}^{\prime}(\tilde{W})-f_{z, c}^{\prime}\left(\tilde{W}-Z_{\alpha}+t\right)\right) K(t, \mathrm{~d} \alpha) \mathrm{d} t, \\
& Q_{3}:=\mathrm{E} \int_{\Gamma} \int_{|t|>1}\left(f_{z, c}^{\prime}\left(\tilde{W}-Z_{\alpha}+t\right)-f_{z, c}^{\prime}(\tilde{W}+t)\right) \hat{K}(t, \mathrm{~d} \alpha) \mathrm{d} t, \\
& Q_{4}:=\mathrm{E} \int_{\Gamma} \int_{|t| \leq 1}\left(f_{z, c}^{\prime}\left(\tilde{W}-Z_{\alpha}+t\right)-f_{z, c}^{\prime}(\tilde{W}+t)\right) \hat{K}(t, \mathrm{~d} \alpha) \mathrm{d} t .
\end{aligned}
$$

It follows from (4.10) that

$$
\begin{aligned}
\left|Q_{2}\right|+\left|Q_{3}\right| & \leq 2 \mathrm{E} \int_{\Gamma} \int_{|t|>1}\left(\mathbf{1}_{\left\{-Y_{\alpha} \leq t<0\right\}}+\mathbf{1}_{\left\{0 \leq t \leq-Y_{\alpha}\right\}}\right)\left|\tilde{H}_{2}(\mathrm{~d} \alpha)\right| \mathrm{d} t \\
& \leq 2 \mathrm{E} \int_{\Gamma}\left|Y_{\alpha}\right| \mathbf{1}_{\left\{\left|Y_{\alpha}\right|>1\right\}}\left|\tilde{H}_{2}(\mathrm{~d} \alpha)\right| \\
& =2 r_{2} .
\end{aligned}
$$

Using (4.11), we obtain

$$
\begin{aligned}
\left|Q_{4}\right| \leq & \mathrm{E} \int_{\Gamma} \int_{|t| \leq 1}(|\tilde{W}|+|t|+1)\left(\left|Z_{\alpha}\right| \wedge 1\right)|\hat{K}(t, \mathrm{~d} \alpha)| \mathrm{d} t \\
& +\frac{1}{c} \mathrm{E} \int_{\Gamma} \int_{|t| \leq 1} \mathbf{1}_{\left\{Z_{\alpha} \geq 0\right\}} \int_{-Z_{\alpha}}^{0} \mathbf{1}_{\{z \leq \tilde{W}+t+u \leq z+c\}} \mathrm{d} u|\hat{K}(t, d \alpha)| \mathrm{d} t \\
& +\frac{1}{c} \mathrm{E} \int_{\alpha \in \Gamma} \int_{|t| \leq 1} \mathbf{1}_{\left\{Z_{\alpha}<0\right\}} \int_{0}^{-Z_{\alpha}} \mathbf{1}_{\{z \leq \tilde{W}+t+u \leq z+c\}} \mathrm{d} u|\hat{K}(t, d \alpha)| \mathrm{d} t \\
\leq & \mathrm{E} \int_{\Gamma}(|\tilde{W}|+1)\left(\left|Z_{\alpha}\right| \wedge 1\right)\left(\left|Y_{\alpha}\right| \wedge 1\right)\left|\tilde{H}_{2}(\mathrm{~d} \alpha)\right| \\
& +\frac{1}{2} \mathrm{E} \int_{\Gamma}\left(\left|Z_{\alpha}\right| \wedge 1\right)\left(Y_{\alpha}^{2} \wedge 1\right)\left|\tilde{H}_{2}(\mathrm{~d} \alpha)\right|+Q_{4,1} \\
\leq & r_{8}+r_{9}+\frac{1}{2} r_{3}+Q_{4,1},
\end{aligned}
$$

where

$$
\begin{aligned}
Q_{4,1}= & \frac{1}{c} \mathrm{E} \int_{\Gamma} \int_{|t| \leq 1} \mathbf{1}_{\left\{Z_{\alpha} \geq 0\right\}} \int_{-Z_{\alpha}}^{0} \mathbf{1}_{\{z \leq \tilde{W}+t+u \leq z+c\}} \mathrm{d} u|\hat{K}(t, \mathrm{~d} \alpha)| \mathrm{d} t \\
& +\frac{1}{c} \mathrm{E} \int_{\Gamma} \int_{|t| \leq 1} \mathbf{1}_{\left\{Z_{\alpha}<0\right\}} \int_{0}^{-Z_{\alpha}} \mathbf{1}_{\{z \leq \tilde{W}+t+u \leq z+c\}} \mathrm{d} u|\hat{K}(t, \mathrm{~d} \alpha)| \mathrm{d} t .
\end{aligned}
$$


Let $\eta(\alpha)=\left.\tilde{H}_{2}\right|_{B_{\alpha}}$. It then follows from Proposition 4.1 that

$$
\begin{aligned}
Q_{4,1}= & \frac{1}{c} \mathrm{E} \int_{\Gamma} \int_{|t| \leq 1} \mathbf{1}_{\left\{Z_{\alpha} \geq 0\right\}} \int_{-Z_{\alpha}}^{0} \mathrm{P}^{\eta(\alpha)}(z \leq \tilde{W}+t+u \leq z+c) \mathrm{d} u|\hat{K}(t, \mathrm{~d} \alpha)| \mathrm{d} t \\
& +\frac{1}{c} \mathrm{E} \int_{\Gamma} \int_{|t| \leq 1} \mathbf{1}_{\left\{Z_{\alpha}<0\right\}} \int_{0}^{-Z_{\alpha}} \mathrm{P}^{\eta(\alpha)}(z \leq \tilde{W}+t+u \leq z+c) \mathrm{d} u|\hat{K}(t, \mathrm{~d} \alpha)| \mathrm{d} t \\
\leq & \left\{\frac{1}{8}\left(4 r_{13}+5\right)+c^{-1}\left[\frac{1}{8}\left(12 r_{13}+17\right) r_{3}+4 r_{2}+2 r_{14}+4 r_{10}\right]\right\} \\
& \times \mathrm{E} \int_{\Gamma} \int_{|t| \leq 1}\left|Z_{\alpha}\right||\hat{K}(t, \mathrm{~d} \alpha)| \mathrm{d} t \\
= & \frac{1}{8}\left(4 r_{13}+5\right) r_{8}+c^{-1}\left[\frac{1}{8}\left(12 r_{13}+17\right) r_{3}+4 r_{2}+2 r_{14}+4 r_{10}\right] r_{8} .
\end{aligned}
$$

Hence,

$$
Q_{4} \leq \frac{1}{8}\left(4 r_{13}+13\right) r_{8}+r_{9}+\frac{1}{2} r_{3}+c^{-1}\left[\frac{1}{8}\left(12 r_{13}+17\right) r_{3}+4 r_{2}+2 r_{14}+4 r_{10}\right] r_{8}
$$

In similar fashion, we obtain

$$
\begin{aligned}
\left|Q_{1}\right| \leq & \mathrm{E} \int_{\Gamma} \int_{|t| \leq 1}(|\tilde{W}|+1) \min \left\{\left|Z_{\alpha}\right|+|t|, 1\right\}|K(t, \mathrm{~d} \alpha)| \mathrm{d} t \\
& +\frac{1}{c} \mathrm{E} \int_{\Gamma} \int_{|t| \leq 1} \mathbf{1}_{\left\{Z_{\alpha} \leq t\right\}} \int_{0}^{t-Z_{\alpha}} \mathbf{1}_{\{z \leq \tilde{W}+u \leq z+c\}} \mathrm{d} u|K(t, \mathrm{~d} \alpha)| \mathrm{d} t \\
& +\frac{1}{c} \mathrm{E} \int_{\Gamma} \int_{|t| \leq 1} \mathbf{1}_{\left\{Z_{\alpha}>t\right\}} \int_{t-Z_{\alpha}}^{0} \mathbf{1}_{\{z \leq \tilde{W}+u \leq z+c\}} \mathrm{d} u|K(t, \mathrm{~d} \alpha)| \mathrm{d} t \\
\leq & \mathrm{E} \int_{\Gamma} \int_{|t| \leq 1}(|\tilde{W}|+1)\left\{\left(\left|Z_{\alpha}\right| \wedge 1\right)+|t|\right\}|K(t, \mathrm{~d} \alpha)| \mathrm{d} t+Q_{1,1} \\
\leq & \mathrm{E} \int_{\Gamma}(|\tilde{W}|+1)\left\{\left(\left|Z_{\alpha}\right| \wedge 1\right)\left(\left|Y_{\alpha}^{*}\right| \wedge 1\right)+\frac{1}{2}\left(\left|Y_{\alpha}^{*}\right|^{2} \wedge 1\right)\right\}\left|\tilde{H}_{2}^{*}(\mathrm{~d} \alpha)\right|+Q_{1,1},
\end{aligned}
$$

where

$$
\begin{aligned}
Q_{1,1}= & \frac{1}{c} \mathrm{E} \int_{\Gamma} \int_{|t| \leq 1} \mathbf{1}_{\left\{Z_{\alpha} \leq t\right\}} \int_{0}^{t-Z_{\alpha}} \mathbf{1}_{\{z \leq \tilde{W}+u \leq z+c\}} \mathrm{d} u|K(t, \mathrm{~d} \alpha)| \mathrm{d} t \\
& +\frac{1}{c} \mathrm{E} \int_{\Gamma} \int_{|t| \leq 1} \mathbf{1}_{\left\{Z_{\alpha}>t\right\}} \int_{t-Z_{\alpha}}^{0} \mathbf{1}_{\{z \leq \tilde{W}+u \leq z+c\}} \mathrm{d} u|K(t, \mathrm{~d} \alpha)| \mathrm{d} t .
\end{aligned}
$$

Since $\mathrm{E}|\tilde{W}| \leq 1$, it follows that, on the one hand,

$$
\begin{aligned}
\left|Q_{1}\right| \leq & \mathrm{E} \int_{\Gamma}(|\tilde{W}|+1)\left(\left|Z_{\alpha}\right| \wedge 1\right)\left(\left|Y_{\alpha}^{*}\right| \wedge 1\right)\left|\tilde{H}_{2}^{*}(\mathrm{~d} \alpha)\right| \\
& +\mathrm{E} \int_{\Gamma}\left(\left|Y_{\alpha}^{*}\right|^{2} \wedge 1\right)\left|\tilde{H}_{2}^{*}(\mathrm{~d} \alpha)\right|+Q_{1,1} \\
= & r_{12}+r_{3}+Q_{1,1}
\end{aligned}
$$


On the other hand, applying Proposition 4.1 gives

$$
\begin{aligned}
Q_{1,1}= & \frac{1}{c} \mathrm{E} \int_{\Gamma} \int_{|t| \leq 1} \mathbf{1}_{\left\{Z_{\alpha} \leq t\right\}} \int_{0}^{-\left(Z_{\alpha}-t\right)} \mathrm{P}^{\eta(\alpha)}(z \leq \tilde{W}+u \leq z+c) \mathrm{d} u|K(t, \mathrm{~d} \alpha)| \mathrm{d} t \\
& +\frac{1}{c} \mathrm{E} \int_{\Gamma} \int_{|t| \leq 1} \mathbf{1}_{\left\{Z_{\alpha}>t\right\}} \int_{-\left(Z_{\alpha}-t\right)}^{0} \mathrm{P}^{\eta(\alpha)}(z \leq \tilde{W}+u \leq z+c) \mathrm{d} u|K(t, \mathrm{~d} \alpha)| \mathrm{d} t \\
\leq & \left\{\frac{1}{8}\left(4 r_{13}+5\right)+c^{-1}\left[\frac{1}{8}\left(12 r_{13}+17\right) r_{3}+4 r_{2}+2 r_{14}+4 r_{10}\right]\right\} \\
& \times \mathrm{E} \int_{\Gamma} \int_{|t| \leq 1}\left(\left|Z_{\alpha}\right|+|t|\right)|K(t, \mathrm{~d} \alpha)| \mathrm{d} t \\
\leq & \left\{\frac{1}{8}\left(4 r_{13}+5\right)+c^{-1}\left[\frac{1}{8}\left(12 r_{13}+17\right) r_{3}+4 r_{2}+2 r_{14}+4 r_{10}\right]\right\} \\
& \times \mathrm{E} \int_{\Gamma}\left\{\left|Z_{\alpha}\right|\left(\left|Y_{\alpha}^{*}\right| \wedge 1\right)+\frac{1}{2}\left(\left|Y_{\alpha}^{*}\right|^{2} \wedge 1\right)\right\}\left|\tilde{H}_{2}^{*}(\mathrm{~d} \alpha)\right| \\
\leq & \left\{\frac{1}{8}\left(4 r_{13}+5\right)+c^{-1}\left[\frac{1}{8}\left(12 r_{13}+17\right) r_{3}+4 r_{2}+2 r_{14}+4 r_{10}\right]\right\}\left(r_{8}^{\prime}+\frac{1}{2} r_{3}\right) .
\end{aligned}
$$

Hence,

$$
\left|Q_{1}\right| \leq r_{3}+r_{12}+\left\{\frac{1}{8}\left(4 r_{13}+5\right)+c^{-1}\left[\frac{1}{8}\left(12 r_{13}+17\right) r_{3}+4 r_{2}+2 r_{14}+4 r_{10}\right]\right\}\left(r_{8}^{\prime}+\frac{1}{2} r_{3}\right) .
$$

Combining (4.12) and the estimates of $Q_{i}, i=1, \ldots, 4$, gives

$$
\begin{aligned}
\sup _{z} \mid & F(z)-\Phi(z) \mid \\
\leq & \frac{1}{5} c+2 r_{2}+\frac{1}{16}\left(29+4 r_{13}\right) r_{3}+\frac{1}{8}\left(4 r_{13}+13\right) r_{8}+\frac{1}{8}\left(4 r_{13}+5\right) r_{8}^{\prime}+r_{9}+r_{12} \\
& +c^{-1}\left(\frac{1}{8}\left(12 r_{13}+17\right) r_{3}+4 r_{2}+2 r_{14}+4 r_{10}\right)\left(r_{8}+r_{8}^{\prime}+\frac{1}{2} r_{3}\right) .
\end{aligned}
$$

Letting

$$
c=\left\{5\left(\frac{1}{8}\left(12 r_{13}+17\right) r_{3}+4 r_{2}+2 r_{14}+4 r_{10}\right)\left(r_{8}+r_{8}^{\prime}+\frac{1}{2} r_{3}\right)\right\}^{1 / 2}
$$

(thus minimizing the right-hand side of (4.13)) and then using $\sqrt{x y} \leq \frac{1}{2}(x+y)$ gives

$$
\begin{aligned}
d_{\mathrm{K}}( & \left.\mathcal{L}\left(\vartheta^{-1}(W-\mathrm{E} W)\right), \mathcal{N}(0,1)\right) \\
\leq & 2 r_{2}+\frac{1}{16}\left(29+4 r_{13}\right) r_{3}+\frac{1}{8}\left(4 r_{13}+13\right) r_{8}+\frac{1}{8}\left(4 r_{13}+5\right) r_{8}^{\prime}+r_{9}+r_{12} \\
& +\frac{1}{5} \sqrt{5}\left\{\left(\frac{1}{8}\left(12 r_{13}+17\right) r_{3}+4 r_{2}+2 r_{14}+4 r_{10}\right)+\left(r_{8}+r_{8}^{\prime}+\frac{1}{2} r_{3}\right)\right\} \\
\leq & 4 r_{2}+\left(3+r_{13}\right) r_{3}+\frac{1}{2}\left(4.2+r_{13}\right) r_{8}+\frac{1}{2}\left(2.2+r_{13}\right) r_{8}^{\prime}+r_{9}+2 r_{10}+r_{12}+r_{14},
\end{aligned}
$$

as claimed in (2.2). The claim in (2.3) is due to the fact that $r_{14} \leq r_{13}$, and, if $r_{13}$ is not less than 1 , the bound becomes obvious.

Proof of Theorem 2.4. Since $\max \left\{\left|Y_{\alpha}\right|,\left|Z_{\alpha}\right|,\left|U_{\alpha}\right|\right\} \leq G\left(N\left(C_{\alpha}\right)\right) / \vartheta$ and $G(\{\alpha\}) / \vartheta \leq$ $G\left(N\left(C_{\alpha}\right)\right) / \vartheta$, we have

$$
\begin{aligned}
& r_{2} \leq \mathrm{E} \int_{\Gamma}\left|Y_{\alpha}\right|^{p-1}\left|\tilde{H}_{2}(\mathrm{~d} \alpha)\right| \leq \eta_{1}, \\
& r_{3} \leq \mathrm{E} \int_{\Gamma}\left|Y_{\alpha}\right|^{p-1}\left|\tilde{H}_{2}(\mathrm{~d} \alpha)\right| \leq \eta_{1}, \\
& r_{8} \leq \mathrm{E} \int_{\alpha \in \Gamma}\left|Y_{\alpha}\right|^{p-2}\left|Z_{\alpha}\right|\left|\tilde{H}_{2}(\mathrm{~d} \alpha)\right| \leq \eta_{1} .
\end{aligned}
$$


We similarly find that $r_{8}^{\prime} \leq \eta_{2}$. By the independence between $\tilde{W}-U_{\alpha}$ and $\left.\tilde{H}_{2}\right|_{B_{\alpha}}$, and since $\left|U_{\alpha}\right| \leq G\left(N\left(C_{\alpha}\right)\right) / \vartheta$, we obtain

$$
\begin{aligned}
r_{9} & \leq \mathrm{E} \int_{\Gamma}\left(\left|\tilde{W}-U_{\alpha}\right|+\left|U_{\alpha}\right|\right)\left(\left|Z_{\alpha}\right| \wedge 1\right)\left|Y_{\alpha}\right|^{p-2} \tilde{H}_{2}(\mathrm{~d} \alpha) \\
& \leq\left(\sup _{\alpha} \mathrm{E}\left|\tilde{W}-U_{\alpha}\right|+1\right) \eta_{1} \\
& \leq\left(\sup _{\alpha} \mathrm{E}\left\{|\tilde{W}|+G\left(N\left(C_{\alpha}\right)\right) / \vartheta\right\}+1\right) \eta_{1} \\
& \leq\left(\sup _{\alpha} \mathrm{E} G\left(N\left(C_{\alpha}\right)\right) / \vartheta+2\right) \eta_{1}, \\
r_{10} & \leq \mathrm{E} \int_{\beta_{1} \in \Gamma} \int_{\beta_{2} \in N\left(A_{\beta_{1}}\right)}\left|Y_{\beta_{1}}\right|^{p-2}\left(\left|\tilde{H}_{2}\left(\mathrm{~d} \beta_{2}\right)\right|+\left|\tilde{H}_{2}^{*}\left(\mathrm{~d} \beta_{2}\right)\right|\right)\left|\tilde{H}_{2}\left(\mathrm{~d} \beta_{1}\right)\right| \\
& \leq \eta_{1}+\eta_{2}
\end{aligned}
$$

and

$$
\begin{aligned}
r_{12} & \leq \mathrm{E} \int_{\alpha \in \Gamma}\left(\left|\tilde{W}-U_{\alpha}\right|+\left|U_{\alpha}\right|+1\right)\left(\left|Z_{\alpha}\right| \wedge 1\right)\left|Y_{\alpha}^{*}\right|^{p-2}\left|\tilde{H}_{2}^{*}(\mathrm{~d} \alpha)\right| \\
& \leq\left(\sup _{\alpha} \mathrm{E}\left|\tilde{W}-U_{\alpha}\right|+2\right) \eta_{2} \\
& \leq\left(\sup _{\alpha} \mathrm{E} G\left(N\left(C_{\alpha}\right)\right) / \vartheta+3\right) \eta_{2}, \\
r_{13} & \leq \sup _{\alpha} \mathrm{E} G\left(N\left(C_{\alpha}\right)\right) / \vartheta,
\end{aligned}
$$

completing the proof, from (2.3), because the bound is obvious if $\sup _{\alpha} \mathrm{E} G\left(N\left(C_{\alpha}\right)\right) / \vartheta>1$.

There is one final technical lemma.

Lemma 4.3. If $Z \sim \operatorname{Po}(\Lambda)$, then, for all $r>0$ and all integers $n \geq \max \{r, 2 \mathrm{e} \Lambda\}$,

$$
\mathrm{E}(Z+1)^{r} \leq n^{r}\left\{1+2.2 \mathrm{e}^{-\Lambda} 2^{-n}\right\} .
$$

Proof. It is immediate that $\mathrm{E}(Z+1)^{r}=\mathrm{E}\left\{(Z+1)^{r} \mathbf{1}_{\{Z<n\}}\right\}+\mathrm{E}\left\{(Z+1)^{r} \mathbf{1}_{\{Z \geq n\}}\right\}$, with the first term on the right-hand side equalling at most $n^{r}$. To bound the second term, just observe, by simple comparison, that, for $n$ in the chosen range,

$$
\begin{aligned}
\sum_{j \geq n}(j+1)^{r} \frac{\mathrm{e}^{-\Lambda} \Lambda^{j}}{j !} & \leq n^{r} \frac{\mathrm{e}^{-\Lambda} \Lambda^{n}}{n !} \sum_{s \geq 0}\left(\frac{n+s+1}{n}\right)^{r}\left(\frac{\Lambda}{n}\right)^{s} \leq n^{r} \frac{\mathrm{e}^{-\Lambda} \Lambda^{n}}{n !} \frac{\mathrm{e}^{r / n}}{1-n^{-1} \Lambda \mathrm{e}^{r / n}} \\
& \leq 2 \mathrm{e}^{r} \frac{\mathrm{e}^{-\Lambda}}{\sqrt{2 \pi n}}\left(\frac{\Lambda \mathrm{e}}{n}\right)^{n},
\end{aligned}
$$

this last from Stirling's formula. The lemma now follows.

\section{Acknowledgements}

The authors wish to thank both the Institute for Mathematical Sciences of the National University of Singapore for their hospitality and support while part of this work was accomplished, and a referee for a number of helpful comments. A. Xia would like to thank the Institut für Mathematik at Universität Zürich for a most enjoyable visit, during which most of the work was done. 


\section{References}

Anscombe, F. J. (1952). Large-sample theory of sequential estimation. Proc. Camb. Phil. Soc. 48, 600-607.

Avram, F. And Bertsimas, D. (1993). On central limit theorems in geometrical probability. Ann. Appl. Prob. 3, 1033-1046.

Bai, Z.-D., ChaO, C.-C., Hwang, H.-K. And Liang, W.-Q. (1998). On the variance of the number of maxima in random vectors and its applications. Ann. Appl. Prob. 8, 886-895.

Bai, Z.-D., Devroye, L., Hwang, H.-K. And Tsai, T.-H. (2005). Maxima in hypercubes. Random Structures Algorithms 27, 290-309.

BAI, Z.-D., HwANG, H.-K., LiAng, W.-Q. AND TsaI, T.-H. (2001). Limit theorems for the number of maxima in random samples from planar regions. Electron. J. Prob. 6, 41 pp.

BALDI, P. And RinotT, Y. (1989). On normal approximations of distributions in terms of dependency graphs. Ann. Prob. 17, 1646-1650.

Barbour, A. D. And Xia, A. (2001). The number of two-dimensional maxima. Adv. Appl. Prob. 33, 727-750.

BARbour, A. D., KARońsKi, M. AND RucińsKi, A. (1989). A central limit theorem for decomposable random variables with applications to random graphs. J. Combinatorial Theory B 47, 125-145.

BARYSHNikov, Yu. (2000). Supporting-points processes and some of their applications. Prob. Theory Relat. Fields 117, 163-182.

Bolthausen, E. (1984). An estimate of the remainder in the combinatorial central limit theorem. Z. Wahrscheinlichkeitsth. 66, 379-386.

Chen, L. H. Y. And Shao, Q.-M. (2004). Normal approximation under local dependence. Ann. Prob. 32, $1985-2028$.

Chen, L. H. Y. And Xia, A. (2004). Stein's method, Palm theory and Poisson process approximation. Ann. Prob. 32, 2545-2569.

Daley, D. J. And Vere-Jones, D. (1988). An Introduction to the Theory of Point Processes. Springer, New York.

Dembo, A. And RinotT, Y. (1996). Some examples of normal approximations by Stein's method. In Random Discrete Structures (IMA Vol. Math. Appl. 76), eds D. Aldous and R. Pemantle, Springer, Berlin, pp. 25-44.

Devroye, L. (1993). Records, the maximal layer, and uniform distributions in monotone sets. Comput. Math. Appl. 25, $19-31$.

Embrechts, P., KlüPpelberg, C. And Mikosch, T. (1997). Modelling Extremal Events. Springer, Berlin.

ERICKSON, R. V. (1974). $L_{1}$ bounds for asymptotic normality of $m$-dependent sums using Stein's technique. Ann. Prob. 2, 522-529.

Gnedenko, B. V. And Korolev, V. Yu. (1996). Random Summation. Limit Theorems and Applications. CRC Press, Boca Raton, FL.

Goovaerts, M. J. And Dhaene, J. (1996). The compound Poisson approximation for a portfolio of dependent risks. Insurance Math. Econom. 18, 81-85.

Kallenberg, O. (1983). Random Measures, 3rd edn. Academic Press, London.

KLÄVER, H. AND Schmitz, N. (2006). An inequality for the asymmetry of distributions and a Berry-Esseen theorem for random summation. J. Inequal. Pure Appl. Math. 7, Article 2, 25 pp.

MAtérn, B. (1986). Spatial Variation (Lecture Notes Statist. 36), 2nd edn. Springer, Berlin.

Penrose, M. D. And Yukich, J. E. (2005). Normal approximation in geometric probability. In Stein's Method and Applications, eds A. D. Barbour and L. H. Y. Chen, Singapore University Press/World Scientific, Singapore, pp. 37-58.

RÉNYI, A. (1960). On the central limit theorem for the sum of a random number of independent random variables. Acta Math. Acad. Sci. Hungar. 11, 97-102.

RinotT, Y. AND Rotar, V. (1996). A multivariate CLT for local dependence with $n^{-1 / 2} \log n$ rate, and applications to multivariate graph related statistics. J. Multivariate Anal. 56, 333-350.

Rolski, T., Schmidli, H., Schmidt, V. And Teugels, J. (1999). Stochastic Processes for Insurance and Finance. John Wiley, Chichester.

SiLvestrov, D. S. (2004). Limit Theorems for Randomly Stopped Stochastic Processes. Springer, London.

SteIN, C. (1972). A bound for the error in the normal approximation to the distribution of a sum of dependent random variables. In Proc. 6th Berkeley Symp. Math. Statist. Prob., Vol. 2, eds L. M. Le Cam, J. Neyman and E. L. Scott, University of California Press, Berkeley, pp. 583-602.

Stein, C. (1986). Approximate Computation of Expectations (IMS Lecture Notes Monogr. Ser. 7). Institute of Mathematical Statistics, Hayward, CA.

Stoyan, D. and Stoyan, H. (1994). Fractals, Random Shapes and Point Fields. Methods of Geometrical Statistics. John Wiley, Chichester. 